This is the Preprint version of our article accepted for publication in Advanced Functional Materials [Advanced Functional Materials, 2105287 (2021)]. The Version of Record is available online: https://doi.org/10.1002/adfm.202105287.

\title{
Controlled chemical functionalization toward 3D-2D carbon nanohorn-MoS 2 heterostructures with enhanced electrocatalytic activity for protons reduction
}

Antonia Kagkoura, ${ }^{1}$ Raul Arenal, ${ }^{2,3,4}$ Nikos Tagmatarchis ${ }^{1 *}$

${ }^{1}$ Theoretical and Physical Chemistry Institute, National Hellenic Research Foundation, 48 Vassileos Constantinou Avenue, 11635 Athens, Greece

${ }^{2}$ Laboratorio de Microscopias Avanzadas (LMA), Universidad de Zaragoza, Mariano Esquillor s/n, 50018 Zaragoza, Spain

${ }^{3}$ Instituto de Nanociencia y Materiales de Aragon (INMA), CSIC-U. de Zaragoza, Calle Pedro Cerbuna 12, 50009 Zaragoza, Spain

${ }^{4}$ ARAID Foundation, 50018 Zaragoza, Spain

E-mail: tagmatar@eie.gr

Keywords: Carbon nanohorns, molybdenum disulfide, heterostructures, functionalization, hydrogen evolution

Abstract: The realization of novel heterostructures arising from the combination of nanomaterials is an effective way to modify their physicochemical and electrocatalytic properties, giving them enhanced characteristics stemming from their individual constituents. Interfacing carbon nanohorns (CNHs) possessing high porosity, large specific surface area and good electrical conductivity, with MoS $_{2}$ owning multiple electrocatalytic active sites but lacking significant conductivity, robust interactions 
and effective structure, can be a strategy to boost the electrocatalytic reduction of protons to molecular hydrogen. Herein, we covalently introduce, in a stepwise approach, complementary functional groups at the conical tips and sidewalls of $\mathrm{CNHs}$, along with the basal plane of $\mathrm{MoS}_{2}$, en route the construction of 3D-2D CNH-MoS 2 heterostructures. The increased $\mathrm{MoS}_{2}$ loading onto $\mathrm{CNHs}$, improving and facilitating charge delocalization and transfer in neighboring $\mathrm{CNHs}$, along with the plethora of active sites, results in excellent electrocatalytic activity for protons reduction same to that of commercial Pt/C. We have registered minute overpotential, low Tafel slope and small chargetransfer resistance for electrocatalyzing the evolution of hydrogen from the newly prepared heterostructure of $0.029 \mathrm{~V}, 71 \mathrm{mV} / \mathrm{dec}$ and $34.5 \Omega$, respectively. Furthermore, the stability of the 3D2D CNH-MoS 2 heterostructure was validated after performing 10,000 ongoing electrocatalytic cycles.

\section{Introduction}

Carbon nanohorns ( $\mathrm{CNHs}$ ) belong to the wider $\mathrm{sp}^{2}$ hybridized carbon nanostructures family, closely related to carbon nanotubes, though with important structural and physical properties differences. To name the most important ones, (a) the tips of $\mathrm{CNHs}$ are conical due to the presence of five-membered rings, (b) CNHs aggregate in 3D spherical superstructures of around $100 \mathrm{~nm}$ in diameter, (c) they are metal-free produced, and (d) they contain external micropores and internal mesopores. ${ }^{1}$ Particularly, the high porosity, large specific surface area, high thermal and chemical stability, good electrical conductivity of $\mathrm{CNHs}$ account for their efficient participation in electrocatalytic reactions, mainly, but not only, as support substrate. ${ }^{2}$ Conversely, although in recent years the inherent insolubility of $\mathrm{CNH}$ in wet media has been overcome thanks to the establishment of diverse functionalization protocols for directing the covalent anchorage of organic species at the tips or sidewalls of $\mathrm{CNHs}^{3-8}$ facilitating their manipulation, hybridization with other nanostructures is scarce. For example, interfacing $\mathrm{CNHs}$ with transition metal dichalcogenides possessing multiple electrocatalytic active sites but lacking significant conductivity, robust interactions and effective structure, toward the formation of novel heterostructures potentially suitable to electrocatalyze, 
among other transformations, protons reduction to molecular hydrogen, e.g. hydrogen evolution reaction (HER), is restricted. ${ }^{9}$

Bonding arrangement, e.g. surface configuration, and size dimension, e.g. reduced thickness from bulk to 2D nanosheets, govern and significantly affect the electronic structure of $\mathrm{MoS}_{2}$ as benchmark transition metal dichalcogenide material. Although $\mathrm{MoS}_{2}$ is found in two polymorphs, the $2 \mathrm{H}$ semiconducting polytype with trigonal prismatic coordinated Mo atoms, and the $1 \mathrm{~T}$ metallic polymorph with octahedral coordination at Mo, the latter one shows intrinsic reduced charge-transfer resistance making it promising for electrocatalysis. ${ }^{10}$ Additionally, unsaturated Mo atoms at the basal plane of $1 \mathrm{~T}-\mathrm{MoS}_{2}$ are electrocatalytically active, opposed to $2 \mathrm{H}-\mathrm{MoS}_{2}$ where catalytically active sites at the basal plane are absent. ${ }^{11}$ In this context, $1 \mathrm{~T}-\mathrm{MoS}_{2}$ has proven to be active in electrocatalyzing protons reduction, especially when hybridized with other species..$^{12-14}$ The latter can be achieved by either in-situ preparation and growth of $1 \mathrm{~T}-\mathrm{MoS}_{2}$ on substrates ${ }^{12,13}$ or by chemical modification of 1T$\mathrm{MoS}_{2 .}{ }^{15}$ Notably, functionalization of $1 \mathrm{~T}-\mathrm{MoS}_{2}$ does not rely on commonly introduced edge-located sulfur vacancies, ${ }^{16,17}$ but mostly rests on electron-rich sulfur species at the basal plane, ${ }^{18,19}$ derived from extended electron-transfer during lithium intercalation upon BuLi exfoliation of the bulk material. Thus, substitution reactions with organic halides and aryl diazonium salts facilitate the incorporation of organic addends onto the basal plane of $1 \mathrm{~T}-\mathrm{MoS}_{2} \cdot{ }^{20-22}$

The combination of different nanostructured materials, forming novel heterostructures, inevitably alters the electronic structure and surface of the interfacing materials and allows overcoming limitations of the individual species, while benefitting from the unique characteristics of the different components. Particularly for heterostructures based on atomically thin 2D nanomaterials featuring unprotected and exposed electrons, noteworthy phenomena such as charge-delocalization and transfer between adjacent nanomaterials, band alignment and enhanced redox properties, occur at the heterointerface. Furthermore, when it comes to heterostructures of $\mathrm{CNHs}$ with $\mathrm{MoS}_{2}$, the unique structure of the latter, containing electropositive and electronegative elements, introduces 
properties, which are generally absent in the former nanostructure, containing only carbon in their framework.

The conceptualization of $\mathrm{CNHs}$, due to their 3D porous network with large surface area, excellent electrical conductivity and high chemical stability, to interface 2D $\mathrm{MoS}_{2}$ nanosheets with metallic phase, for electrocatalyzing protons reduction en route hydrogen generation, is of paramount importance and has yet to be realized. To further boost and advance the electrocatalytic properties of such 3D-2D heterostructures, conjugation of increased amount of 1T-MoS 2 , carrying active catalytic centers for HER, onto CNHs is required. Herein, by employing advanced functionalization methodologies, we initially covalently attached $\mathrm{MoS}_{2}$ nanosheets at the tips of $\mathrm{CNH}$ and then further incorporated additional $\mathrm{MoS}_{2}$ at the sidewalls of CNHs, to realize robust CNH-MoS with high $\mathrm{MoS}_{2}$ loading. We carefully followed every modification step and verified the success of performed reactions by FT-IR and Raman spectroscopy together with thermogravimetry and electron microscopy imaging means. As proof-of-concept, the electrocatalytic activity of the 3D-2D heterostructured CNH-MoS nanoconjugate toward protons reduction was assessed by linear sweep voltammetry and electrochemical impedance spectroscopy and found that the increased $\mathrm{MoS}_{2}$ loading onto $\mathrm{CNHs}$ benefits the process. Markedly, the covalent linkage between $\mathrm{CNHs}$ and $\mathrm{MoS}_{2}$ improves chargetransfer to the catalytic sites between heterointerfaces and delivers greater interfacial surface area

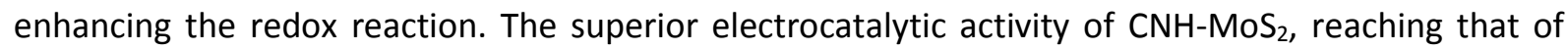
commercial Pt/C, while exhibiting excellent stability and durability, highlights the potentiality of such 3D-2D heterostructures as vital components for fuel cell technologies and/or production of renewable fuels.

\section{Results and discussion}

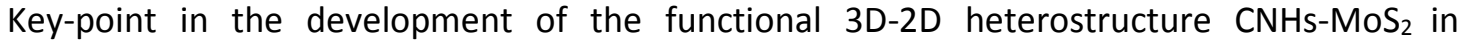
electrocatalysis is the covalent linkage between the two nanostructured materials. Considering that neither $\mathrm{CNHs}$ nor $\mathrm{MoS}_{2}$ contain dangling bonds, activation by incorporation of suitable linkers carrying 
complementary functional groups, at the conical tips and sidewalls of $\mathrm{CNHs}$ as well as at the basal plane of $\mathrm{MoS}_{2}$, is required. In addition, the covalent interactions tightly holding together CNHs and $\mathrm{MoS}_{2}$, compared to the weaker supramolecular or van der Waals ones, significantly perturb their electronic properties within the heterostructure. Furthermore, they also provide structural local matching, between $\mathrm{MoS}_{2}$ and the conical nanotubules forming the aggregated secondary superstructure of $\mathrm{CNHs}$, in the 2D direction. All these, together with the intrinsic high porosity of $\mathrm{CNHs}$, enhance the density of the electrocatalytic active sites within the 3D-2D heterostructure CNHs-MoS2, facilitating protons reduction as it is shown in this work.

The realization of 3D-2D heterostructure $\mathrm{CNH}_{\mathrm{N}}-\mathrm{MoS}_{2}$, with increased $\mathrm{MoS}_{2}$ loading onto $\mathrm{CNH}$, is based on a controlled stepwise functionalization route. For this purpose, separately $\mathrm{MoS}_{2}$ and CNHs were pre-modified so that to introduce appropriate organic functional units for precisely connecting the two species via robust covalent bonding. In more detail, starting with the pre-modification of 2D

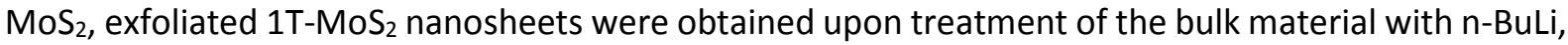
followed by hydration and aqueous sonication of the intercalated species. ${ }^{21}$ Subsequently, $1 \mathrm{~T}-\mathrm{MoS}_{2}$ was reacted with in-situ generated diazonium salts ${ }^{21,22}$ derived from aniline derivative 1 to yield $\mathrm{MoS}_{2-}$ based material 2. Treatment of the latter with trifluoracetic acid, followed by neutralization of the adduct by triethylamine, produced amino-modified $\mathrm{MoS}_{2}$ nanosheets $\mathbf{3}$ (Scheme $\mathbf{1}$ ). The amino-loading of $\mathbf{3}$ was calculated by the Kaiser test and found to be $100 \mu \mathrm{mol} / \mathrm{g}$. On a separate functionalization route, the basal plane of exfoliated 1T-MoS 2 nanosheets was decorated with carboxylic acid units to yield material 4, via reaction of $1 \mathrm{~T}-\mathrm{MoS}_{2}$ with in-situ generated diazonium salts derived from 4-(4aminophenyl)butyric acid. Then, continuing with the pre-modification of $\mathrm{CNHs}$, light-assisted oxidation with hydrogen peroxide, ${ }^{23}$ furnished oxidized $\mathrm{CNH}$ 5 5 , featuring carboxylic acid moieties at their conical tips. Next, with those properly modified $\mathrm{MoS}_{2}$ nanosheets $\mathbf{3}$ and $\mathrm{CNHs} \mathbf{5}$ in hand, having complementary functional units for directing their coupling, we proceeded with the conjugation. Condensation reaction between amino-modified $\mathrm{MoS}_{2} 3$ and oxidized CNHs 5 yielded hybrid 6 (Scheme 1). The amino-loading of 6 was dropped to $15 \mu \mathrm{mol} / \mathrm{g}$ (Kaiser test), verifying the success of the 
condensation reaction. In order to increase the loading of $1 \mathrm{~T}-\mathrm{MoS}_{2}$ onto $\mathrm{CNHs}$ in the heterostructure, we performed a second functionalization reaction onto the sidewalls of $\mathrm{CNHs}$ participating in conjugate 6. Specifically, reaction of in-situ generated azomethine ylides, ${ }^{6}$ derived from the thermal condensation reaction between tert-butyloxycarbonyl (BOC)-protected $\alpha$-amino acid $\mathbf{7}$ and formaldehyde, followed by acidic deprotection of the BOC moiety and subsequent neutralization, yielded heterostructure $\mathbf{8}$, featuring doubly modified $\mathrm{CNHs}_{\text {carrying }} \mathrm{MoS}_{2}$ at the tips and fused pyrrolidine rings at the sidewalls being $\mathrm{N}$-alkylated and terminated to amine groups. The aminoloading of 8 was calculated by the Kaiser test and found to be $80 \mu \mathrm{mol} / \mathrm{g}$. At this point, condensation of those free amine groups with the carboxylic acid units present in modified $\mathrm{MoS}_{2}$ nanosheets 4 resulted on additional incorporation of $\mathrm{MoS}_{2}$, this time at the sidewalls of $\mathrm{CNHs}$, yielding heterostructure 9 (Scheme 1). Again, the drop of amino-loading in 9, being $15 \mu \mathrm{mol} / \mathrm{g}$ (Kaiser test), guarantees the efficiency of the condensation reaction. 


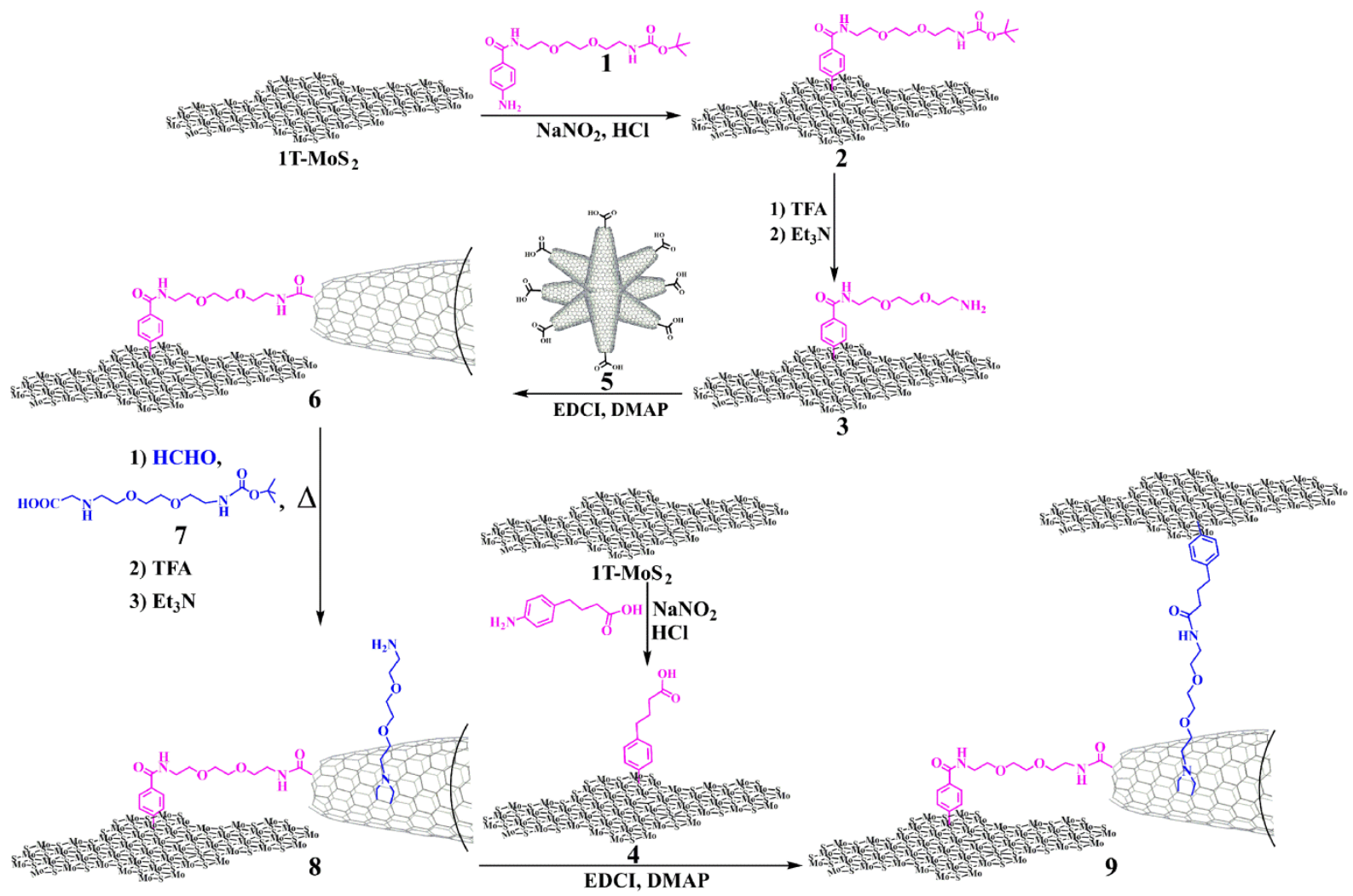

Scheme 1. Synthetic illustration of the preparation of $\mathrm{CNH}-\mathrm{MoS}_{2}$ heterostructure.

In order to follow every different modification occurred on $\mathrm{MoS}_{2}$ and $\mathrm{CNHs}$, with ultimate aim to verify

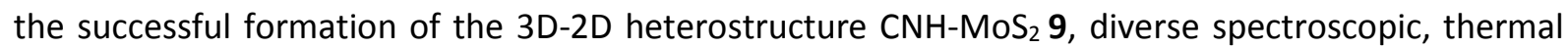
and microscopy imaging techniques were employed. Specifically, the FT-IR spectrum of BOC-modified $\mathrm{MoS}_{2}$ material 2 is controlled by observing the characteristic bands due to carbonyl stretching of benzamide and BOC protecting group at $1645 \mathrm{~cm}^{-1}$ and $1691 \mathrm{~cm}^{-1}$, respectively, while alkyl C-H vibrational features are discernable in the range $2840-2980 \mathrm{~cm}^{-1}$ (Supporting Information, Figure S1). Markedly, the carbonyl BOC band is absent in the FT-IR spectrum of amino-modified $\mathrm{MoS}_{2}$ nanosheets 3, where particularly vibrations only due to the carbonyl stretching of the benzamide at $1645 \mathrm{~cm}^{-1}$ are evident. On the other hand, the FT-IR spectrum of oxidized CNHs $\mathbf{5}$ is occupied by the characteristic carbonyl vibration mode at $1705 \mathrm{~cm}^{-1}$ due to the surface $-\mathrm{COOH}$ units (Supporting Information, Figure S2). Heterostructure 9 shows enhanced the band at $1640 \mathrm{~cm}^{-1}$, as compared to that observed for 6 
(Figure 1a), due to the increased carbonyl amides, e.g. derived by condensation and subsequent incorporation of additional $\mathrm{MoS}_{2}$ species at the sidewalls of CNHs.
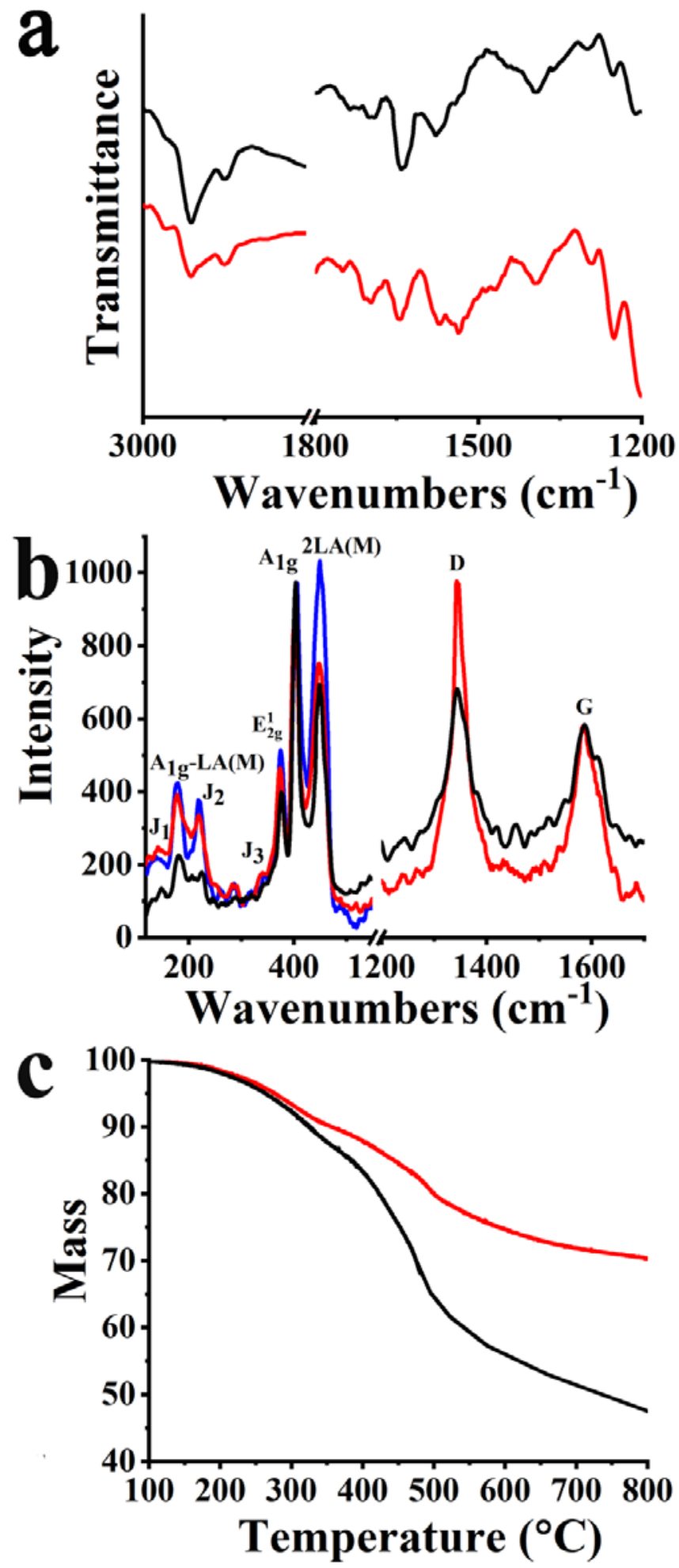

Figure 1. (a) ATR-IR spectra for 6 (red) and $\mathrm{CNH}_{-}-\mathrm{MoS}_{2}$ heterostructure 9 (black). (b) Raman spectra for

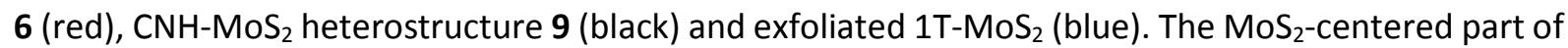


the spectrum $\left(120-550 \mathrm{~cm}^{-1}\right)$ is recorded upon excitation at $633 \mathrm{~nm}$, while the $\mathrm{CNH}$-based part of the spectrum (1200-1700 $\left.\mathrm{cm}^{-1}\right)$ is recorded upon excitation at $514 \mathrm{~nm}$. The spectra shown at the $1200-$ $1700 \mathrm{~cm}^{-1}$ area are normalized at the G-band and their intensity is enhanced compared to the $\mathrm{MoS}_{2^{-}}$ centered part of the spectrum (120-550 $\mathrm{cm}^{-1}$ ) for clarity. (c) TGA graphs for 6 (red) and CNH-MoS heterostructure 9 (black), obtained under nitrogen atmosphere.

Additional insight on the formation of $\mathrm{CNHs}^{-\mathrm{MoS}_{2}}$ heterostructure 9 was acquired by Raman spectroscopy. By employing on-resonance excitation $(633 \mathrm{~nm})$, with respect to the energy of the Aexciton, ${ }^{24}$ and adjusting the laser power to $0.3 \mathrm{~mW} / \mathrm{cm}^{2}$ and exposure to 10 seconds to avoid overheating of the samples, we examined all modified $\mathrm{MoS}_{2}$-based materials. Specifically, the spectrum of exfoliated 1T-MoS 2 is governed by the $A_{1 g}-L A(M), E^{1}{ }_{2 g}, A_{1 g}$ and $2 L A(M)$ Raman active modes at $177,373,404$ and $450 \mathrm{~cm}^{-1}$, respectively. In addition, $J_{1}, J_{2}$ and $J_{3}$ bands located at 145, 220 and 340 $\mathrm{cm}^{-1}$, respectively, are fingerprint phonon modes of the metallic $1 \mathrm{~T}$ octahedral phase of $\mathrm{MoS}_{2}$ (Figure 1b). Notably, from those $J$ phonon modes the $J_{2}$ is the most intense one, and discernible in all modified $\mathrm{MoS}_{2}$-based materials 2-4 (Supporting Information, Figure S3), proving the stabilization of the octahedral 1T-MoS 2 phase upon aryl diazonium salts functionalization, in agreement with earlier studies. ${ }^{15,20}$ Moreover, the intensity ratio $2 \mathrm{LA}(\mathrm{M}) / \mathrm{A}_{1 \mathrm{~g}}$ can be used to assess the basal plane functionalization of $1 \mathrm{~T}-\mathrm{MoS}_{2} \cdot{ }^{21}$ Specifically, the $2 \mathrm{LA}(\mathrm{M}) / \mathrm{A}_{1 \mathrm{~g}}$ intensity ratio for materials $\mathbf{2 - 4}$ appeared decreased to ca. 0.75 due to the basal plane chemical modification as compared to the value of 1.07 for exfoliated 1T-MoS (Supporting Information, Figure S3). The particular ratio remained unchanged not only after the removal of the BOC group in material 3 , but also in hybrid $\mathbf{6}$, since conjugation of $1 \mathrm{~T}$ $\mathrm{MoS}_{2}$ at the tips of oxidized CNHs 5 has no further impact on the basal plane of the $\mathrm{MoS}_{2}$ nanosheets (Figure 1b).

Additionally, in order to determine the functionalization degree in CNHs graphitic network, Raman spectroscopy was performed upon excitation at $514 \mathrm{~nm}$. The Raman spectrum of oxidized CNHs 5 is dominated by the characteristic D- and G- bands at 1340 and $1592 \mathrm{~cm}^{-1}$, related to defects and the 
$\mathrm{sp}^{2}$ graphitic network, respectively. Most importantly, alterations of the intensity of the D-band are strong indicators of the functionalization degree in $\mathrm{CNHs} .{ }^{25}$ The $\mathrm{D} / \mathrm{G}$ intensity ratio was increased to 1.44 for oxidized CNHs 5 (Supporting Information, Figure S4) compared to the value of 1.14 for pristine CNHs due to the generation of defected sites at CNHs tips. ${ }^{23}$ This value was found unchanged for hybrid 6 since the conjugation of $1 \mathrm{~T}-\mathrm{MoS}_{2}$ at the tip of oxidized $\mathrm{CNHs} 5$ does not induce any further alteration on the graphitic network (Supporting Information, Figure S4). On the opposite, heterostructure 8 showed an increase of the $I_{D} / I_{G}$ ratio, ca. 1.55 , due to the second covalent functionalization resulting to new defected sites at the sidewalls of $\mathrm{CNHs}$ (Supporting Information, Figure S4). Markedly, the $\mathrm{I}_{\mathrm{D}} / \mathrm{G}$ ratio remained the same for $\mathrm{CNH}-\mathrm{MoS}_{2}$ heterostructure $\mathbf{9}$, considering that the interface of additional $\mathrm{MoS}_{2}$ nanosheets occurs via conjugation on the already grafted organic chains present at the sidewalls of CNHs (Figure 1b).

The impact of functionalization on the lattice disorder of $\mathrm{MoS}_{2}$ is further corroborated by performing spatial Raman mapping measurements and accordingly probing the $2 \mathrm{LA}(\mathrm{M}) / \mathrm{A}_{1 \mathrm{~g}}$ ratio. In this context, Raman maps (633 nm) were constructed by acquiring and collecting 120 point-spectra (30 $\mu \mathrm{m} \times 30 \mu \mathrm{m}$ area) for 1T-MoS and modified $\mathrm{MoS}_{2}$-based materials 2, 3 and 6 (Figure 2) as well as 4 (Supporting Information, Figure S5). The average $2 \mathrm{LA}(\mathrm{M}) / \mathrm{A}_{1 \mathrm{~g}}$ ratio for $\mathbf{2 - 4}$ and $\mathbf{6}$ is by around $30 \%$ lower as compared to that of exfoliated $1 \mathrm{~T}-\mathrm{MoS}_{2}$ due to symmetry disorder and proving enhancement of the local strain to basal plane modification. Analogously, the structural characteristics of $\mathrm{CNHs}$ component in heterostructure 9 were elucidated by Raman mapping assays ( $514 \mathrm{~nm}$ ) by collecting 120 point-spectra (30 $\mu \mathrm{m} \times 30 \mu \mathrm{m}$ area) and compared to pristine CNHs at Figure 3. The corresponding Raman maps for materials $\mathbf{5 - 8}$, for evaluating the defects induced at the lattice as a result of the chemical modification, are shown at the Supporting Information, Figure S6. The average D/G ratio for oxidized CNHs 5, ca. 1.44, is by around $\sim 21 \%$ higher as compared to that of pristine CNHs, ca. 1.14, resulted from the incorporation of $-\mathrm{COOH}$ units at the tips of $\mathrm{CNHs}$. Meanwhile, for 8 and 3D-2D heterostructure 9 , the average $D / G$ ratio, ca. 1.55 , is by around $\sim 7 \%$ higher as compared to that of 6 , ca. 1.45 , due to disorder of the graphitic network brought by hybridization change from $\mathrm{sp}^{2}$ to $\mathrm{sp}^{3}$ as 
resulted by the additional grafting of addends and subsequent interface of $\mathrm{MoS}_{2}$ at the sidewalls of CNHs.
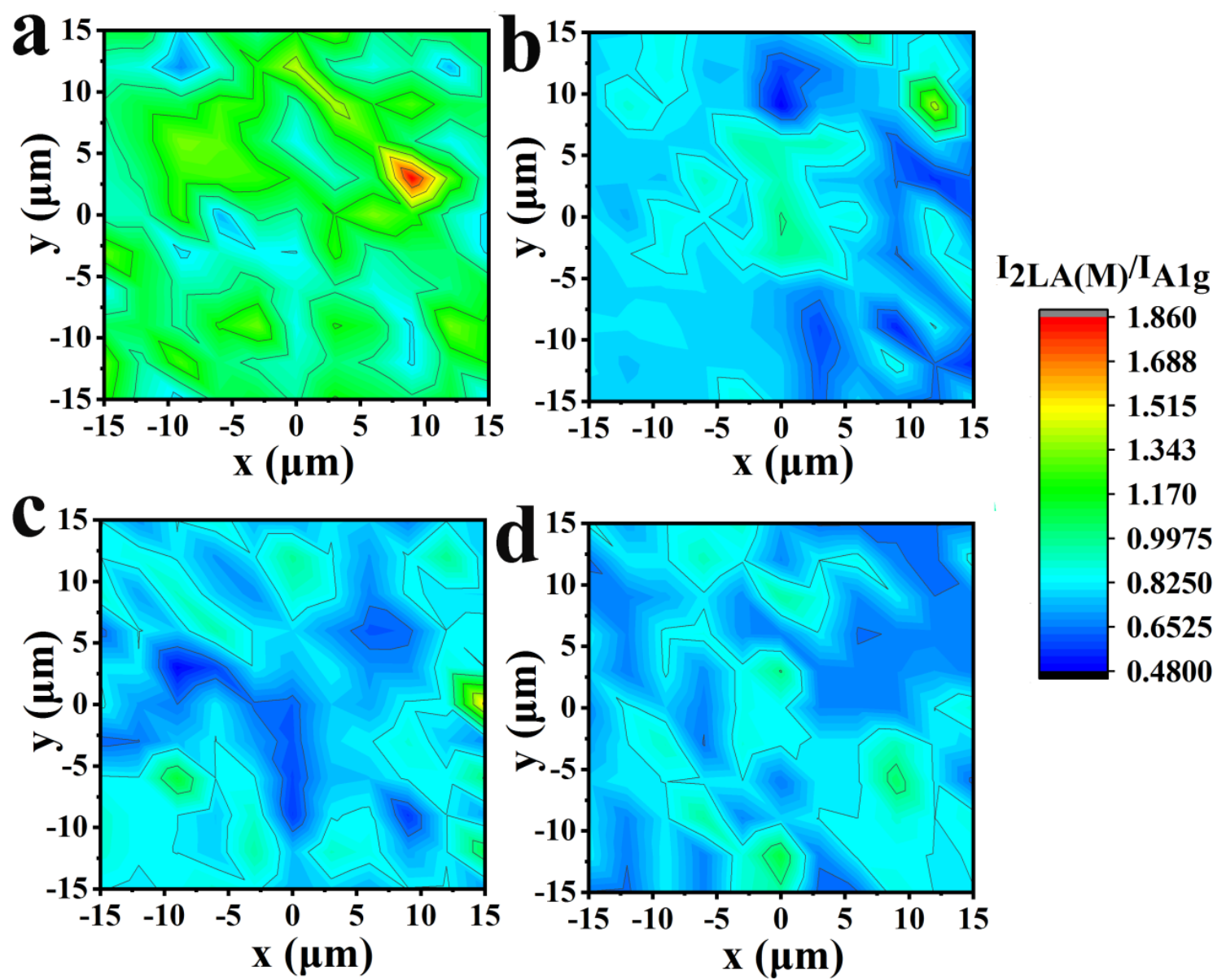

0.4800

Figure 2. Raman maps of (a) exfoliated 1T-MoS $\mathrm{MoS}_{2}$ material 3, and (d) material 6, recorded upon excitation at $633 \mathrm{~nm}$. 

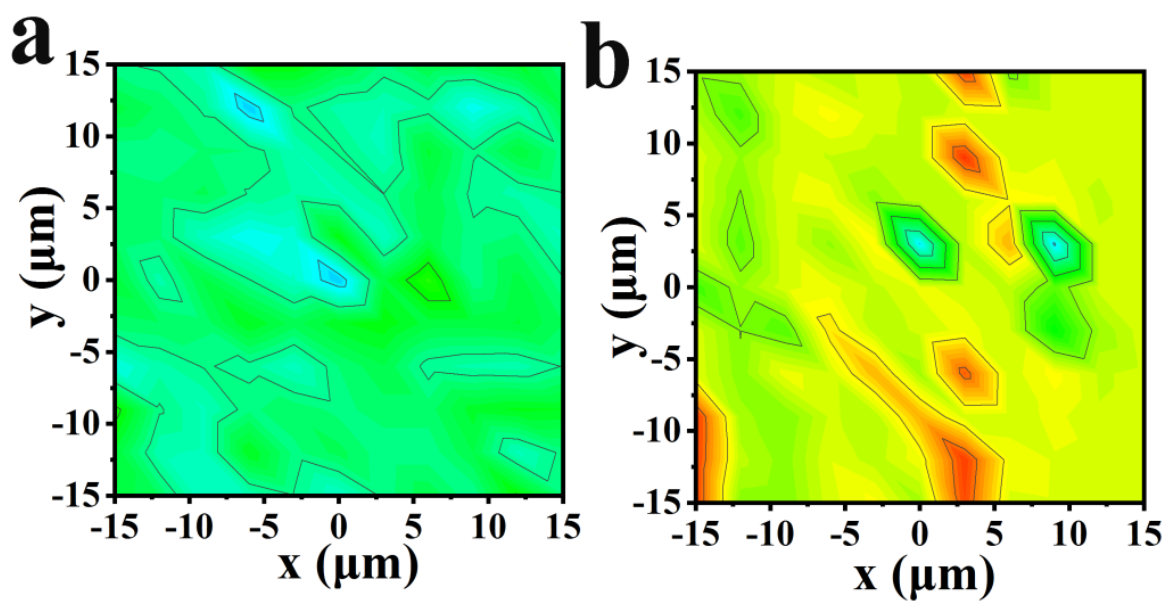

ID/IG

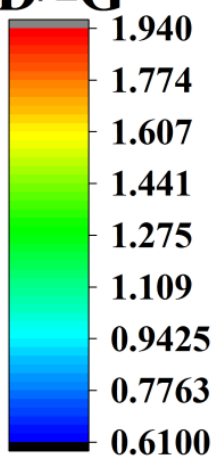

Figure 3. Raman maps of (a) pristine CNHs, and (b) 3D-2D heterostructure 9 recorded upon excitation at $514 \mathrm{~nm}$.

XPS was also employed to get additional information about the chemistry performed and identify the atomic species corresponding to the addends that link $\mathrm{CNHs}_{\text {and }} \mathrm{MoS}_{2}$ in heterostructure 9. In more detail, Figure 4a shows a survey XPS spectrum for the $\mathrm{CNH}-\mathrm{MoS}_{2}$ material 9, where the existence of $\mathrm{C}, \mathrm{Mo}, \mathrm{S}$ and $\mathrm{O}$ is confirmed. High-resolution spectra of these elements have been acquired and also depicted in this figure. The S $2 p$ core-level spectrum is shown in Figure $4 b$, where two strong peaks at 162.0 and $163.2 \mathrm{eV}$ are observed. These peaks correspond to $S 2 p_{3 / 2}$ and $S 2 p_{1 / 2}$ binding energies for $\mathrm{S}^{2-}$. Figure $4 \mathrm{c}$ displays the XPS spectrum in the region of the $\mathrm{S} 2 \mathrm{~s}$ and Mo $3 \mathrm{~d}$ orbitals. The deconvolution of the different peaks discerned in this spectrum is as follows: the peak at $226.3 \mathrm{eV}$ corresponds to S $2 \mathrm{~s}$ and is assigned to $\mathrm{MoS}_{2}$ nanosheets; the second peak at $229.0 \mathrm{eV}$ corresponds to $\mathrm{Mo}^{4+} 3 \mathrm{~d}_{5 / 2}$; the third peak at $\sim 232.3 \mathrm{eV}$ is the contribution of two different configurations of molybdenum, those of $\mathrm{Mo}^{4+} 3 \mathrm{~d}_{3 / 2}$ and $\mathrm{Mo}^{6+} 3 \mathrm{~d}_{3 / 2}$; the last peak at $235.6 \mathrm{eV}$ is due to $\mathrm{Mo}^{6+} 3 \mathrm{~d}_{5 / 2 .}$. Thus, two different molybdenum contributions can be detected, namely, $\mathrm{Mo}^{4+}$ representing around $90 \%$ of the total amount and assigned to $\mathrm{MoS}_{2}$ (as expected in the $1 \mathrm{~T}$ phase), ${ }^{26}$ and $\mathrm{Mo}^{6+}$ of around $10 \%$ corresponding to $\mathrm{MoO}_{3}$. This small amount of oxide suggests that during the different chemical transformations and/or treatments developed on these materials, some oxygen is incorporated in the outermost layer of $\mathrm{MoS}_{2}$, hindering further diffusion to the flakes and protecting them from undesired deeper oxidation. It is important to point out that the quantitative analysis of the $\mathrm{S} / \mathrm{Mo}^{4+}$ (equal to 
1.99) in the Mo 3d S 2s region confirms the good stoichiometry for $\mathrm{MoS}_{2}$ within heterostructure 9.

Figure $4 \mathrm{~d}$ displays the $\mathrm{C} 1 \mathrm{~s}$ part of the XPS spectrum, with the peaks at 284.5, $284.9,286.2$ and 288.1 $\mathrm{eV}$ corresponding to $\mathrm{C}=\mathrm{C}$ and $\mathrm{C}-\mathrm{C}, \mathrm{COOH}$ and $\mathrm{C}=\mathrm{O}$, respectively. ${ }^{27}$ Finally, in Figure $4 \mathrm{e}$, the $\mathrm{N} 1 \mathrm{~s}$ contribution at $399.9 \mathrm{eV}$ has been observed, confirming the presence of nitrogen in the linkers that join the two nanostructures in $\mathrm{CNH}-\mathrm{MoS}_{2}$ material 9.
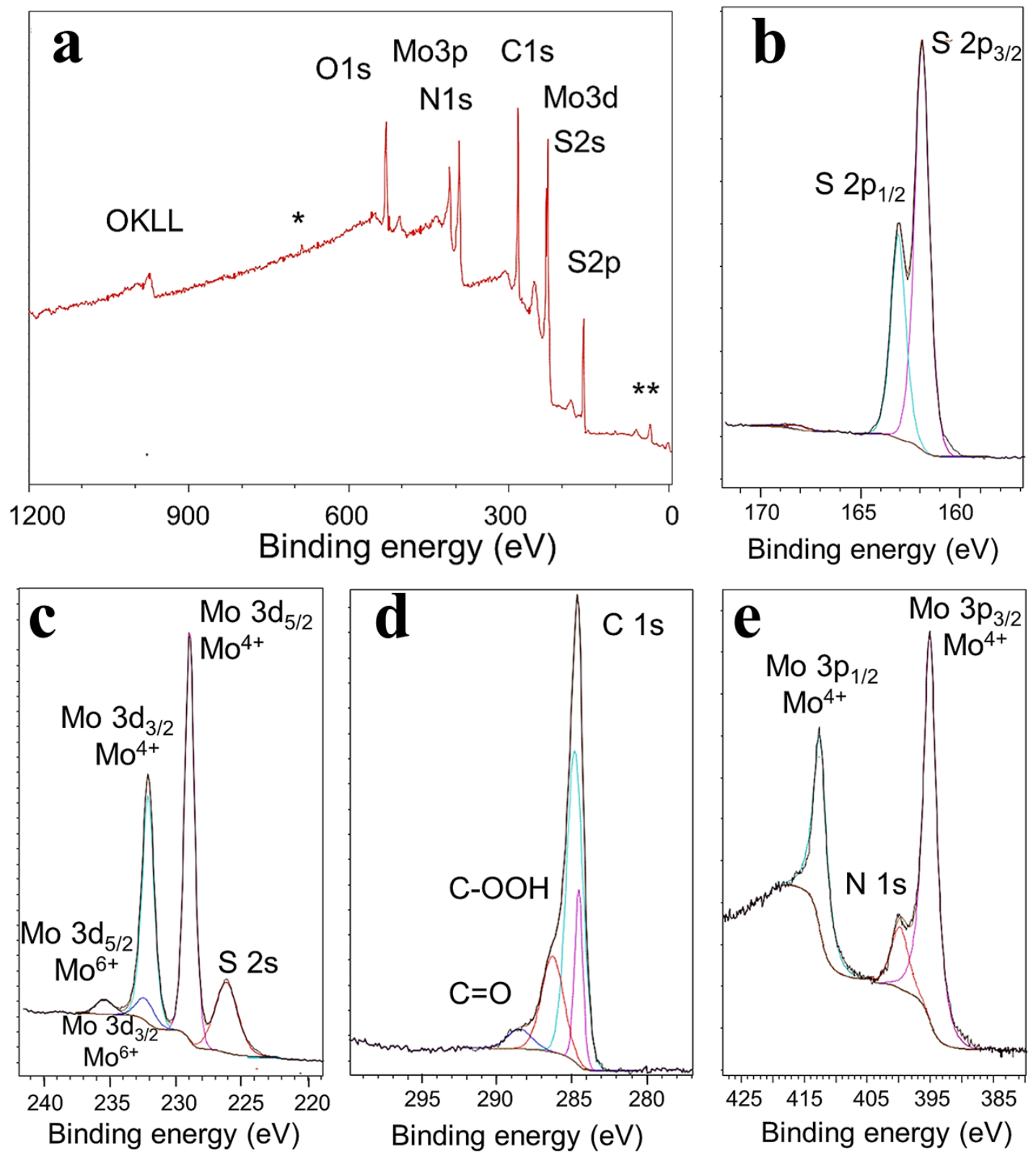

Figure 4. (a) XPS survey spectrum of $\mathrm{CNH}_{-} \mathrm{MoS}_{2}$ heterostructure 9 , where $S 2 p, S 2 s$, Mo 3d, C 1s, N 1s, Mo $3 p$ and $\mathrm{O} 1$ s are observed. Impurities $<1 \%$ corresponding to $\mathrm{F}$ and $\mathrm{Si}$ are shown with * and **, 
respectively. (b-e) High-resolution XPS spectra of $\mathrm{CNH}^{-\mathrm{MoS}_{2}}$ heterostructure 9 , recorded on the same area, and corresponding to S 2p, S 2s-Mo 3d, C 1s and Mo $3 p-N$ 1s, respectively.

Structural and chemical analyses, at the subnanometer scale, have been developed via different (scanning) transmission electron microscopy ((S)TEM) techniques, see Figure 5. The combination of different TEM techniques allow to perform detailed analysis of these nanostructures. ${ }^{12,}$ 14, 16, 23, 28 Figure 5a,b display two low magnification micrographs corresponding to $\mathrm{MoS}_{2}$ nanosheets and to the heterogeneous system of $\mathrm{CNHs}$ and nanosheets in 9. These images show how both nanostructures are organized. It should be mentioned that TEM observations confirm the highest abundance of $\mathrm{MoS}_{2}$ with respect to CNHs. These nanomaterials can be clearly observed in the HRTEM images of Figure 5c-e; see also Supporting Information, Figure S7 for images corresponding to hybrid material 8 as well as exfoliated $\mathrm{MoS}_{2}$. As above mentioned (see Scheme 1), the $\mathrm{MoS}_{2}$ nanosheets are seen both anchored at the tips to $\mathrm{CNHs}$ as well as incorporated on their sidewalls. Figure $5 \mathrm{f}$ corresponds to a HAADF-STEM image of one of these nanostructures confirming what it was observed by HRTEM (Figure 5c-5e). In the white rectangular region marked in that image of Figure 5f, a STEMEELS spectrum-image was acquired. Figure $5 \mathrm{~g}$ shows a combination of chemical maps of two of the elements detected in that area, $\mathrm{C}$ and $\mathrm{S}$, which correspond to $\mathrm{CNHs}$ and to $\mathrm{MoS}_{2}$, respectively. 


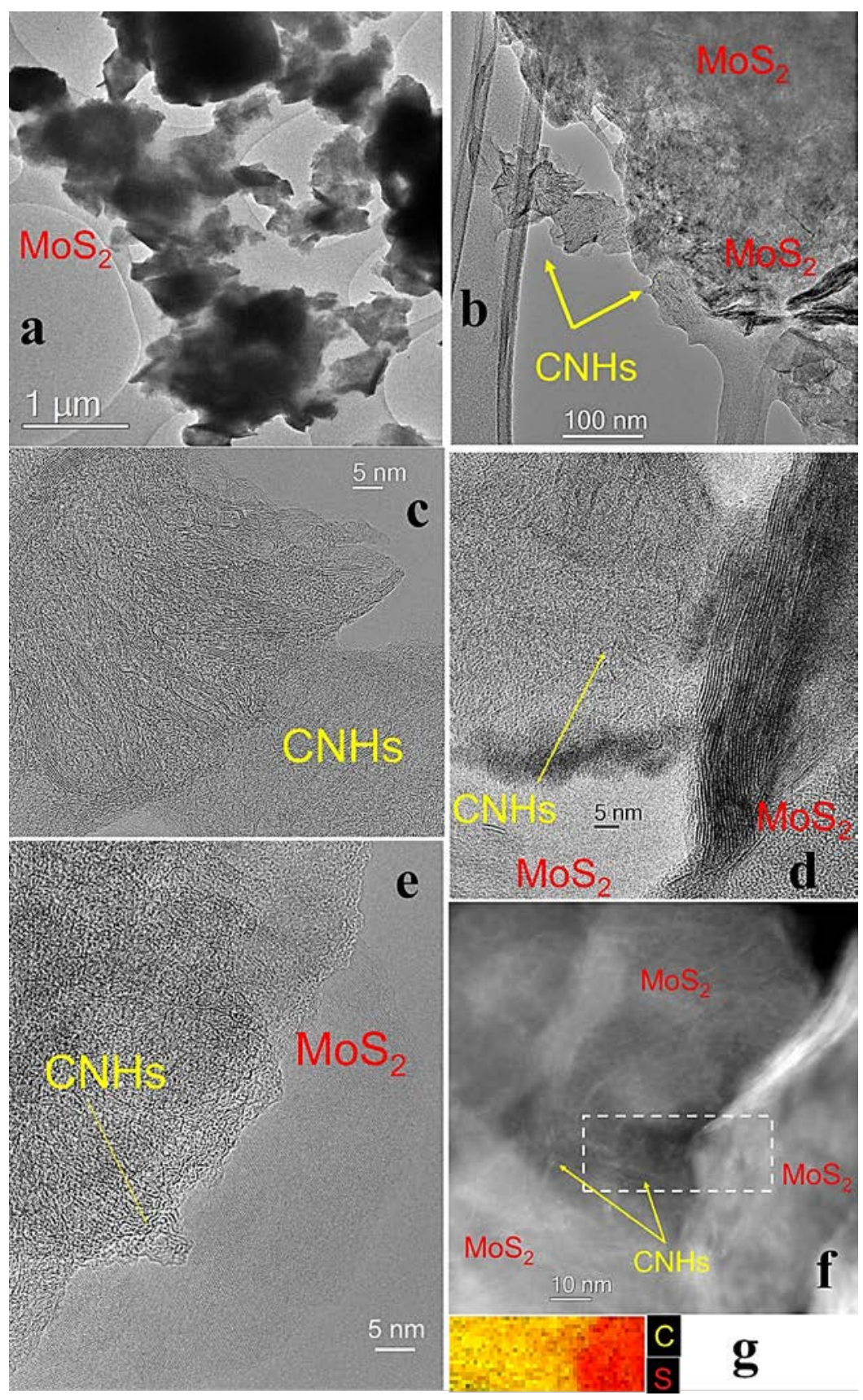

Figure 5. (a, b) Low-magnification TEM micrographs of ${\mathrm{CNH}-M o S_{2}}_{2}$ heterostructure 9, where (a) $\mathrm{MoS}_{2}$ nanosheets and (b) hybrid nanostructures (CNHs and $\mathrm{MoS}_{2}$ flakes) can be observed respectively. (c-e) HRTEM micrographs displaying the distribution of the two constituents of these heterostructures, namely CNHs and the $\mathrm{MoS}_{2}$ nanosheets. (f) HAADF-STEM image showing one of these CNH-MoS heterostructures. A STEM-EELS spectrum-image has been recorded in the rectangular dashed white area highlighted in Fig. 5f. (g) The combined colour chemical map showing $\mathrm{C}$ (in yellow; from the $\mathrm{CNHs}$ ) and S (in red; corresponding to the $\mathrm{MoS}_{2}$ nanosheets) distribution. 
For collecting information about the thermal stability of heterostructure 9 and all intermediate modification steps for $\mathrm{CNHs}$ and $\mathrm{MoS}_{2}$, as well as estimating the amount of organic units covalently anchored onto the 3D and 2D nanostructures, thermogravimetric analysis (TGA) under $\mathrm{N}_{2}$ atmosphere was performed. In this context, exfoliated $1 \mathrm{~T}-\mathrm{MoS}_{2}$ presents a $4 \%$ mass loss up to $500{ }^{\circ} \mathrm{C}$ due to the presence of defects related to the preparation method, ${ }^{21}$ while BOC-modified $\mathrm{MoS}_{2}$ material 2 shows a higher mass loss of $16 \%$ up to same temperature region due to the thermal decomposition of the incorporated organic species (Supporting Information, Figure S8). This percentage is lower for the amino-modified $\mathrm{MoS}_{2}$ material3, ca. 7\%, as expected, due to the absence of the BOC protecting group that contributes to the system with higher molecular weight. Based on that, the loading in the aminomodified $\mathrm{MoS}_{2}$ material 3 was calculated to be one functional group for every $15 \mathrm{MoS}_{2}$ units. Meanwhile, the thermograph of material 4 exhibits a $10 \%$ mass loss up to $500{ }^{\circ} \mathrm{C}$, also corresponds to 1 functional unit per every $15 \mathrm{MoS}_{2}$ units (Supporting Information, Figure S8). At the same time, pristine $\mathrm{CNHs}$ present an almost stable thermal profile under nitrogen atmosphere up to $800{ }^{\circ} \mathrm{C}$, while oxidized CNHs 5 lose $9 \%$ of mass up to $500^{\circ} \mathrm{C}$, due to the thermal decomposition of the carboxylic acid groups (Supporting Information, Figure S9). The thermal decomposition at higher temperature is ascribed to the gradual destruction of the graphitic skeleton, occurring at $\mathrm{sp}^{3}$ hybridized sites, where functionalization took place. In addition, the thermograph of material 6 reveals a higher mass loss of $27 \%$ up to $500{ }^{\circ} \mathrm{C}$, showing the successful hybridization of oxidized $\mathrm{CNHs}^{5}$ with modified $\mathrm{MoS}_{2}$ material 3 (Figure 1c). The subsequent second modification of $\mathrm{CNHs}$ at the sidewalls via the addition of in-situ generated azomethine ylides is screened with a mass loss of $25 \%$ up to $500{ }^{\circ} \mathrm{C}$ for heterostructure 8 (Supporting Information, Figure S8). Lastly, the increased mass loss of $46 \%$ in the thermograph of heterostructure 9 compared to that of $\mathbf{6}$ (Figure 1c) advocates the successful addition of $\mathrm{MoS}_{2}$ nanosheets onto the sidewalls of $\mathrm{CNHs}$.

In order to validate the aspirations of our aim, we constructed pseudoelectrodes by dropcasting dispersions of the 3D-2D heterostructure 9 onto a glassy-carbon electrode and assessed their activity for protons reduction in a proof-of-concept application. Markedly, comparing the 
electrocatalytic behavior of 9 with that of 6 toward HER, the beneficial role of higher loading of $\mathrm{MoS}_{2}$ nanosheets, covalently anchored at the sidewalls of the doubly-modified CNHs, was revealed. In more detail, performing linear sweep voltammetry (LSV) assays in aqueous $0.5 \mathrm{M} \mathrm{H}_{2} \mathrm{SO}_{4}$ electrolyte, an onset overpotential at 0.029 V vs RHE was recorded for $\mathrm{CNH}^{-M_{0}} \mathrm{~S}_{2}$ heterostructure $\mathbf{9}$ (Figure 6a), which is by $249 \mathrm{mV}$ lower than the one recorded for 6 , ca. $-0.220 \mathrm{~V}$ vs RHE. Notably, the excess energy above the needed thermodynamic potential to drive protons reduction to molecular hydrogen, i.e. onset overpotential, for $\mathbf{9}$ is same with the one noted for $\mathrm{Pt} / \mathrm{C}$ employed as reference material, highlighting the advantageous nature of the newly prepared heterostructure. Furthermore, considering that the functional current density required for electrochemical water splitting and sufficient hydrogen production is $-10 \mathrm{~mA} / \mathrm{cm}^{2}$, while misinterpretations from inherent electroactivity can be avoided at that value, it is reasonable to use the potential required to reach the aforementioned current density as point of reference for protons reduction by $\mathbf{9}$ and compare it with that of $\mathbf{6}$. Specifically, while for $\mathbf{6}$ an overpotential of $-0.340 \mathrm{~V}$ vs RHE was registered at $-10 \mathrm{~mA} / \mathrm{cm}^{2}$ current density, the 3D-2D heterostructure 9 operates the HER at $-0.028 \mathrm{~V}$ vs RHE, ca. $312 \mathrm{mV}$ lower than that of 6 , and close to the overpotential registered for the reference Pt/C at -0.009 V vs RHE (Figure 6a). The significantly lower overpotential value for driving protons reduction to molecular hydrogen with heterostructure 9 over $\mathbf{6}$ is directly related to the presence of increased electrocatalytic centers and clearly mirrors the higher loading of $\mathrm{MoS}_{2}$ nanosheets at both the tips and sidewalls of CNHs. The superior electrocatalytic activity of heterostructure $\mathbf{9}$ toward protons reduction is ascribed to the electronic communication between the two covalently linked species within the 3D-2D nanoconjugate and the plethora of $\mathrm{MoS}_{2^{-}}$ based active centers incorporated onto the extended $\pi$-electronic conductive network of CNHs. The latter is further corroborated by considering the significantly inferior HER electrocatalytic activity of heterostructures prepared by direct growth of $\mathrm{MoS}_{2}$ onto graphene $\mathrm{e}^{12,13,29-32}$ or via electrostatic interactions between $\mathrm{MoS}_{2}$ and graphene. ${ }^{33}$ From the LSVs of $\mathrm{MoS}_{2}$-based material 3, exfoliated 1T$\mathrm{MoS}_{2}$ and oxidized CNHs 5 (Supporting Information, Figure S10a), higher overpotential values compared to that of $\mathbf{9}$ for HER are noted. The slightly weakened electrocatalytic activity of $\mathbf{3}$ versus 
exfoliated 1T-MoS 2 is rationalized by considering the removal/neutralization of negative charges from the surface of $1 \mathrm{~T}-\mathrm{MoS}_{2}$ due to the functionalization and formation of covalent $\mathrm{S}-\mathrm{C}$ bonds. ${ }^{15}$

Insight on the reaction kinetics and the exchange current for the electrocatalytic protons reduction with heterostructure 9 was obtained by the Tafel slope extracted from the corresponding LSV curve. Briefly, in electrochemical kinetics, the Tafel slope relates the rate of HER to the overpotential and can give useful information about how quickly the current increases with the applied overpotential. With the above in mind, heterostructure 9 possesses a low Tafel slope value of 71 $\mathrm{mV} / \mathrm{dec}$ (Figure 6b), which manifests that hydrogen production is rate-limited by the electrochemical desorption of adsorbed hydrogen atoms onto the modified pseudoelectrode. On the contrary, 6 exhibits a high Tafel slope value of $161 \mathrm{mV} / \mathrm{dec}$ (Figure 6b), revealing different rate-limiting step for HER, namely adsorption of protons onto the pseudoelectrode surface via a reduction process. The Tafel slope values for $\mathrm{MoS}_{2}$-based material 3, exfoliated 1T-MoS 2 and oxidized $\mathrm{CNHs}^{5}$ (Supporting Information, Figure S10b) are also high, ca. 150, 153 and $274 \mathrm{mV} / \mathrm{dec}$, respectively.

Additional insight on HER kinetics is obtained by electrochemical impedance spectroscopy (EIS) assays. The EIS measurements were conducted at a potential where significant HER current was recorded, corresponding to $-2 \mathrm{~mA} / \mathrm{cm}^{2}$, while EIS data were fitted to Randles circuit. Briefly, the low frequency region in EIS was employed to determine the charge-transfer resistance $\left(R_{c t}\right)$ at the interface with the electrolyte. Thus, Nyquist plot showed the smallest frequency semicircle for heterostructure 9, attributed to small $R_{\mathrm{ct}}$ of $34.5 \Omega$ (Figure $6 \mathrm{c}$ ) as a result of high conductance derived by the covalently incorporated CNHs in the 3D-2D heterostructure. On the contrary, 6 showed $\mathrm{R}_{\mathrm{ct}}$ of $79.0 \Omega$, higher than the value calculated for exfoliated 1T-MoS 2 at $57.0 \Omega$ (Supporting Information, Figure S10c). The higher conductance of 6 compared to exfoliated 1T-MoS 2 , as deduced from EIS and the calculated $R_{c t}$ values, is rationalized by considering the removal/neutralization of the negative charges in $\mathrm{MoS}_{2}$ due to the aryl diazonium salts functionalization leading to lower conductance. In fact, this is validated by examining the $R_{c t}$ of modified $M_{0} S_{2}$ nanosheets 3 , calculated to be $72.0 \Omega$, similar to that of 6 and 
higher than that of exfoliated $1 \mathrm{~T}-\mathrm{MoS}_{2}$ due to lower conductance resulted from the quenching of the negative surface charge upon chemical modification. ${ }^{15}$ Overall, the results from EIS as presented by the calculated $R_{c t}$ agree with the HER kinetics and Tafel slope values for the efficient performance of heterostructure 9 .

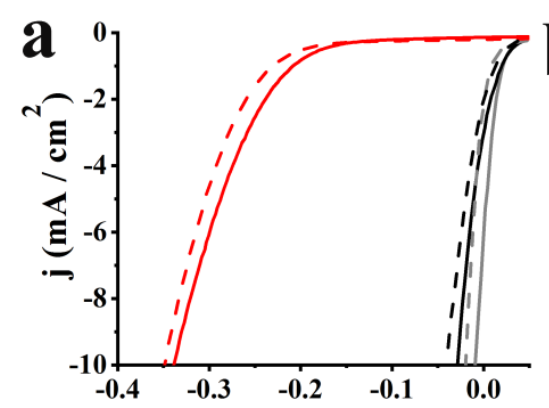

E / V vs. RHE
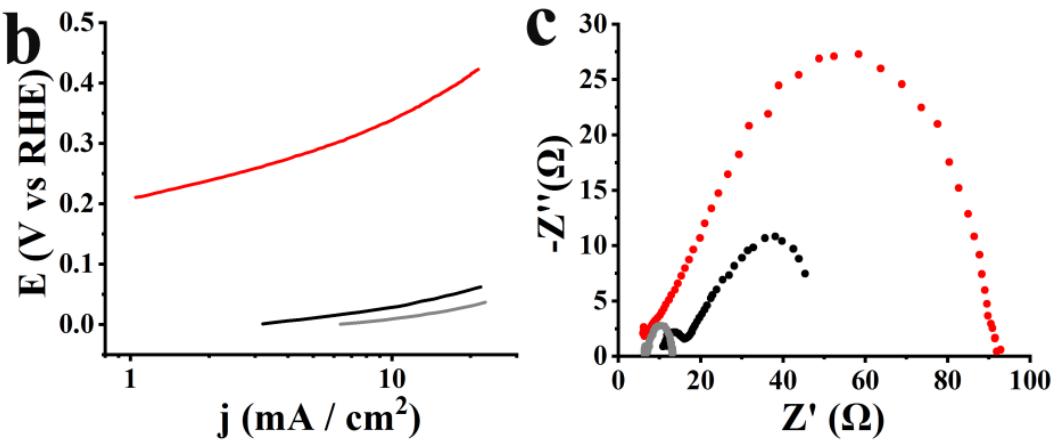

Figure 6. (a) LSVs for HER before (solid lines) and after 10,000 cycles (dashed lines), (b) Tafel slopes, and (c) Nyquist plots, for materials 6 (red), 9 (black) and Pt/C (grey). The LSVs obtained at 1,600 rpm rotation speed and $5 \mathrm{mV} / \mathrm{s}$ scan rate in aqueous $0.5 \mathrm{M} \mathrm{H}_{2} \mathrm{SO}_{4}$.

The stability of 9 was assessed, after performing 10,000 ongoing electrocatalytic cycles. Specifically, heterostructure 9 proved to be extremely stable after 10,000 cycles by exhibiting a negatively shifted LSV curve of $11 \mathrm{mV}$ (Figure 6a). The same was noticed for Pt/C employed as reference. On the contrary, 6 as well as exfoliated 1T-MoS 2 , modified $\mathrm{MoS}_{2}$ nanosheets $\mathbf{3}$ and oxidized $\mathrm{CNHs} \mathbf{5}$ showed higher overpotentials of 40-90 mV after continues cycling (Supporting Information, Figure S10a). Table 1 summarizes the electrochemical HER data before and after 10,000 cycles for heterostructure 9 and compares with those of $\mathbf{6}$ as well as exfoliated $1 \mathrm{~T}-\mathrm{MoS}_{2}$ and oxidized CNHs 5. 
Table 1. Electrocatalytic HER parameters for heterostructure 9 in comparison with materials 3, 6, oxidized CNHs 5, exfoliated 1T-MoS 2 and Pt/C.

\begin{tabular}{|c|c|c|c|c|}
\hline Electrocatalyst & $\begin{array}{l}\text { Onset potential } \\
\qquad \text { (V vs RHE) }\end{array}$ & $\begin{array}{l}\text { Potential (V vs RHE) at } \\
-10 \mathrm{~mA} / \mathrm{cm}^{2}\end{array}$ & $\begin{array}{l}\text { Tafel slope } \\
\text { (mV/dec) }\end{array}$ & $\mathbf{R}_{\mathrm{ct}}(\Omega)$ \\
\hline Heterostructure 9 & 0.029 & -0.028 & 71 & 34.5 \\
\hline $\begin{array}{c}\text { Heterostructure } \\
\qquad 9^{\mathrm{a}}\end{array}$ & 0.02 & -0.039 & 67 & - \\
\hline $\mathrm{Pt} / \mathrm{C}$ & 0.029 & -0.009 & 35 & 6.1 \\
\hline $\mathrm{Pt} / \mathrm{C}^{\mathrm{a}}$ & 0.011 & -0.020 & 35 & - \\
\hline 6 & -0.22 & -0.34 & 161 & 79 \\
\hline $6^{a}$ & -0.25 & -0.43 & 209 & - \\
\hline $\begin{array}{l}\text { Exfoliated 1T- } \\
\qquad \mathrm{MoS}_{2}\end{array}$ & -0.16 & -0.28 & 153 & 57.0 \\
\hline $\begin{array}{l}\text { Exfoliated 1T- } \\
\mathrm{MoS}_{2}{ }^{\mathrm{a}}\end{array}$ & -0.21 & -0.32 & 150 & - \\
\hline 3 & -0.18 & -0.31 & 150 & 72 \\
\hline $3^{a}$ & -0.25 & -0.40 & 165 & - \\
\hline oxidized CNHs 5 & -0.25 & -0.43 & 274 & 197.0 \\
\hline oxidized CNHs $5^{\text {a }}$ & -0.29 & -0.48 & 240 & - \\
\hline
\end{tabular}

Finally, in order to further assess the beneficial role of covalently linking the species composing the $\mathrm{CNH}-\mathrm{MoS}_{2}$ heterostructure, we evaluated the electrocatalytic HER activity of pseudoelectrodes fabricated by physical mixing materials 3 and 5 as well as 4 and 6 , abbreviated as mixtures $A$ and $B$ respectively, and compare them with materials 6 and 9, respectively. As expected from the LSVs of the mixed materials (Supporting Information, Figure S11a), extremely higher onset potential values for HER, ca. -0.41 and $-0.19 \mathrm{~V}$ vs RHE and potential values at $-10 \mathrm{~mA} / \mathrm{cm}^{2} \mathrm{ca} .-0.66$ and $-0.35 \mathrm{~V}$ vs RHE 
compared to that of 6 and 9 ca. -0.22 and $0.029 \mathrm{Vvs}$ RHE and ca. -0.34 and $-0.028 \mathrm{~V}$ vs RHE, respectively, are noted. The poor hydrogen production is also mirrored by the extremely high Tafel slope values i.e. 415 and 211 for mixtures $A$ and $B$, respectively, versus 161 and 71 for 6 and 9, respectively, highlighting the sluggish current flow within the species (Supporting Information, Figure S11b) due to inadequate and weak interactions. Lastly, the stability of the mixtures was assessed, after performing 10,000 ongoing electrocatalytic cycles. Unsurprisingly, mixtures A and B showed higher onset overpotentials by ca. 272 and $520 \mathrm{mV}$ and potentials registered at $-10 \mathrm{~mA} / \mathrm{cm}^{2} \mathrm{ca} .280$ and $681 \mathrm{mV}$ as compared to the values registered for 6 and 9, respectively, after continues cycling (Supporting Information, Figure S11a) due to the absence of strong interactions holding tight the species forming the heterostructures. Overall, the above findings underline the importance of the development of heterostructures, wherein components are firmly bound together, i.e. via covalent linkage, in order to promote charge transfer and current flow but also to ensure stability; all of them being crucial factors for electrocatalysis.

\section{Conclusions}

The combination of nanomaterials with interesting characteristics in the form of advanced heterostructures is a potent approach to boost their properties and overtake limitations that individual components might face. In this work, we demonstrated a novel functionalization approach towards the preparation of the 3D-2D CNH-MoS 2 heterostructure, relying on the modification of the conical tips and sidewalls of $\mathrm{CNHs}$ to covalently link with chemically transformed $\mathrm{MoS}_{2}$ nanosheets. We carefully followed every modification step and verified the success of performed reactions by FT-IR and Raman spectroscopy, XPS, TGA, and electron microscopy imaging and spectroscopy means. The plethora of catalytic active sites due to the $\mathrm{MoS}_{2}$ incorporation in the 3D modified porous $\mathrm{CNH}$ superstructure and the covalent linkage between $\mathrm{CNH}$ and $\mathrm{MoS}_{2}$ improving charge-transfer at the heterointerfaces enhanced the electrocatalytic properties of the $\mathrm{CNH}-\mathrm{MoS}_{2}$ heterostructure, which showed excellent activity toward protons reduction, same as the one noted for commercial Pt/C. Our results are fully supported by data acquired from linear sweep voltammetry and electrochemical 
impedance spectroscopy, highlighting minute overpotential, low Tafel slope and small charge-transfer resistance for electrocatalyzing the evolution of hydrogen. Furthermore, the stability of the $\mathrm{CNH}-\mathrm{MoS}_{2}$ heterostructure was validated after performing 10,000 ongoing electrocatalytic cycles. All in all, this advanced functionalization approach towards the preparation of the novel 3D-2D heterostructure may open new routes for the realization and exploration of functional heterostructures.

\section{Experimental Section/Methods}

General. Chemicals, reagents, and solvents were purchased from Sigma-Aldrich and used without further purification. For the light-assisted oxidation reaction of $\mathrm{CNH}$ s the light source used was a 500 W halogen lamp, which was positioned $20 \mathrm{~cm}$ away from the reactor. Infrared (IR) spectra were obtained on a Fourier Transform IR spectrometer (Equinox 55 from Bruker Optics) equipped with a single reflection diamond ATR accessory (DuraSamp1IR ॥ by SensIR Technologies). Raman measurements were recorded with a Renishaw confocal spectrometer at 514 and $633 \mathrm{~nm}$. The data were obtained and analysed with Renishaw Wire and Origin software. Thermogravimetric analysis was acquired using a TGA Q500 V20.2 Build 27 instrument by TA in a nitrogen (purity $>99.999 \%$ ) inert atmosphere. HRTEM studies have been carried out in an image-corrected corrected FEI Titan-Cube 60300 working at $80 \mathrm{kV}$. Scanning transmission electron microscopy (STEM) and electron-energy loss spectroscopy (EELS) analyses have been developed in a probe-corrected FEI Titan-Low-Base 60-300 operating at $80 \mathrm{kV}$ (equipped with a X-FEG ${ }^{\circledR}$ gun and Cs-probe corrector (CESCOR from CEOS GmbH)). EELS studies have been performed using the spectrum-image mode. ${ }^{34,35}$ The different $\mathrm{CNH}^{3} \mathrm{MoS}_{2}$ powders have been dispersed in ethanol and the suspensions have been ultrasonicated and dropped onto copper carbon holey grids. XPS data were acquired using a Kratos Axis Supra spectrometer equipped with a monochromated AI Ka X-ray source using an analyzer pass energy of $160 \mathrm{eV}$ for survey spectra and $20 \mathrm{eV}$ for the core level spectra. Spectra were recorded by setting the instrument to the hybrid lens mode and the slot mode providing approximately a $700 \times 300 \mu \mathrm{m}^{2}$ analysis area using charge neutralization. Regions have been calibrated using the reference value $\mathrm{BE}(\mathrm{C} 1 \mathrm{~s} \mathrm{sp} 2)=284.5 \mathrm{eV}$. 
All XPS spectra were analyzed using CASA XPS software. The XPS peaks were fitted to GL(70) Voigt lineshape (a combination of $70 \%$ Gaussian and 30\% Lorentzian character), after performing a Shirley background subtraction. All electrochemical measurements were carried out using an Autolab PGSTAT128N potentiostat/galvanostat and were carried out at room temperature in a standard threecompartment electrochemical cell by using a graphite rod as a counter-electrode, an RDE with glassy carbon disk (geometric surface area: $\left.0.0196 \mathrm{~cm}^{2}\right)$ as a working electrode, and $\mathrm{Hg} / \mathrm{HgSO}_{4}\left(0.5 \mathrm{M} \mathrm{K}_{2} \mathrm{SO}_{4}\right)$ as reference electrode. LSV measurements for HER were carried out at room temperature in $\mathrm{N}_{2}$ saturated aqueous $0.5 \mathrm{M} \mathrm{H}_{2} \mathrm{SO}_{4}$. The catalyst ink was prepared by dispersing $4.0 \mathrm{mg}$ of the catalytic powder in a $1 \mathrm{~mL}$ mixture of deionized water, isopropanol, and 5\% Nafion ( $/ \mathrm{v} / \mathrm{v}=4: 1: 0.02)$ and sonicated for 30 min prior use. Before casting the electrocatalytic ink on the electrode's surface, the working electrode was polished with 6, 3 and $1 \mathrm{~mm}$ diamond pastes, rinsed with deionized water, and sonicated in double-distilled water. Afterwards, $8.5 \mu \mathrm{L}$ aliquots of the electrocatalyst were casted on the electrode surface and were left to dry at room temperature. Electrochemical impedance spectroscopy (EIS) measurements were conducted from $10^{5}$ to $10^{-1} \mathrm{~Hz}$ with an AC amplitude of $0.01 \mathrm{~V}$.

Exfoliated 1T-MoS 2 . In a round-bottom flask, bulk $\operatorname{MoS}_{2}(1.5 \mathrm{gr})$ was treated with n-butyllithium (20 $\mathrm{mL}, 2.5 \mathrm{M}$ in hexane) and allowed to stir under an inert atmosphere for $48 \mathrm{~h}$ at $70^{\circ} \mathrm{C}$. The reaction was then quenched with the slow addition of $\sim 100 \mathrm{~mL}$ of distilled water. After hydrogen evolution ceased, deionized water $(150 \mathrm{~mL})$ was added and the dispersion was bath sonicated for 1 hour, transferred to a beaker and left overnight to settle. Afterwards, the supernatant was collected in order to avoid nonexfoliated $\mathrm{MoS}_{2}$. The dispersion was filtrated through PTFE membrane filter $(0.2 \mu \mathrm{m})$ and extensively washed with deionized water and hexane. Next, the filter cake was dispersed in methanol and centrifuged at $4000 \mathrm{rpm}$ for 5 minutes ( 5 times) with methanol to obtain $300 \mathrm{mg}$ of $1 \mathrm{~T}-\mathrm{MoS}_{2}$ as powder.

MoS $_{2}$-based material 2. In a round-bottom flask, exfoliated $1 \mathrm{~T}-\mathrm{MoS}_{2}(160 \mathrm{mg}$ ) was dissolved in deionized $\mathrm{H}_{2} \mathrm{O}(160 \mathrm{~mL}$ ) and sonicated for $30 \mathrm{~min}$. In a separate round-bottom flask, aniline derivative $1(1.0 \mathrm{gr}, 2.7 \mathrm{mmol})$ was dissolved in a mixture of deionized $\mathrm{H}_{2} \mathrm{O}(32 \mathrm{~mL})$ and concentrated $\mathrm{HCl}(0.4$ 
$\mathrm{mL}$ ). Then, $\mathrm{NaNO}_{2}(789 \mathrm{mg}, 11.4 \mathrm{mmol})$ was slowly added in the solution under vigorous stirring at 0 ${ }^{\circ} \mathrm{C}$ and $20 \% \mathrm{HCl}(2.4 \mathrm{~mL})$. The reaction was left for 45 minutes to stir. Afterwards, the solution was added in the $1 \mathrm{~T}-\mathrm{MoS}_{2}$ dispersion at $0{ }^{\circ} \mathrm{C}$ under stirring and a black precipitate was formed. The reaction mixture was left to stir for $2 \mathrm{~h}$ at $0{ }^{\circ} \mathrm{C}$ and then for another $4 \mathrm{~h}$ at ambient. The resulting suspension was filtrated through PTFE membrane filter (pore size $0.2 \mu \mathrm{m}$ ) and washed with deionized $\mathrm{H}_{2} \mathrm{O}$, methanol and acetone to yield $150 \mathrm{mg}$ of $\mathbf{2}$ as powder.

MoS $_{2}$-based material 3. Material $2(150 \mathrm{mg})$ was dispersed in dichloromethane $(100 \mathrm{~mL})$ and treated with trifluoroacetic acid ( $5 \mathrm{~mL}$ ), while the dispersion was left to stir overnight. After filtration in a PTFE membrane filter $(0.2 \mu \mathrm{m})$ and washing with deionized water, acetone and methanol, the filter cake was dispersed in methanol $(50 \mathrm{~mL})$. Then, triethylamine $(10 \mathrm{~mL})$ was added and the dispersion was left to stir for 15 minutes. Finally, the dispersion was filtered with PTFE membrane filter $(0.2 \mu \mathrm{m})$ and washed with dimethylformamide, acetone and methanol. The solid residue was collected to obtain $150 \mathrm{mg}$ of $\mathbf{3}$ as powder.

MoS $_{2}$-based material 4. In a round bottom flask, $1 \mathrm{~T}-\mathrm{MoS}_{2}(120 \mathrm{mg})$ was dissolved in deionized $\mathrm{H}_{2} \mathrm{O}$ $(120 \mathrm{~mL})$ and the suspension was sonicated for $30 \mathrm{~min}$. In a separate round-bottom flask, commercially available 4-(4-aminophenyl)butyric acid $(350 \mathrm{mg}, 2 \mathrm{mmol})$, was dissolved in a mixture of deionized $\mathrm{H}_{2} \mathrm{O}$ $(25 \mathrm{~mL})$ and concentrated $\mathrm{HCl}(0.3 \mathrm{~mL})$. Then, $\mathrm{NaNO}_{2}(135 \mathrm{mg}, 1.95 \mathrm{mmol})$ was slowly added in the solution under vigorous stirring at $0{ }^{\circ} \mathrm{C}$ and $20 \% \mathrm{HCl}(1.9 \mathrm{~mL})$ was added and the reaction was left under stirring for 45 minutes at $0{ }^{\circ} \mathrm{C}$. Afterwards, the solution was added in the $1 \mathrm{~T}-\mathrm{MoS}_{2}$ dispersion at $0{ }^{\circ} \mathrm{C}$ under stirring and a black precipitate was formed. The reaction was left to stir for $2 \mathrm{~h}$ at $0{ }^{\circ} \mathrm{C}$ and then for another $4 \mathrm{~h}$ at ambient. The resulting suspension was filtrated through PTFE membrane filter (pore size $0.2 \mu \mathrm{m}$ ) and washed with deionized water, methanol and acetone to obtain $120 \mathrm{mg}$ of $\mathbf{4}$ as powder.

Oxidized CNHs 5. In a round-bottom flask, pristine CNHs (30 mg) were treated with $\mathrm{H}_{2} \mathrm{O}_{2}(30 \%, 50 \mathrm{~mL})$ under light irradiation at $120^{\circ} \mathrm{C}$ for 3 hours. Then, the dispersion was filtrated through PTFE membrane 
filter (pore size $0.2 \mu \mathrm{m}$ ) and the solid residue was extensively washed with deionized water and methanol to obtain $30 \mathrm{mg}$ of $\mathbf{5}$ as powder.

CNH-based material 6. In a two-neck round bottom flask, material $5(2 \mathrm{mg})$ was added in dry dichloromethane $\left(15 \mathrm{~mL}\right.$ ) and the suspension was bath sonicated for 30 min under $\mathrm{N}_{2}$ atmosphere. Afterwards, 1-ethyl-3-(3-dimethylaminopropyl)carbodiimide $(5.13 \mathrm{mg}, 0.027 \mathrm{mmol})$ was added at $0{ }^{\circ} \mathrm{C}$ and the resulting suspension was left to stir for $1 \mathrm{~h}$. Then, $1 \mathrm{~T}-\mathrm{MoS}_{2}(14.5 \mathrm{mg})$, 4-dimethylaminopyridine (4.7 mg, $0.0382 \mathrm{mmol}$ ) and $\mathrm{N}, \mathrm{N}$-diisopropylethylamine $(20 \mu \mathrm{L})$ were added and the dispersion was left to stir for five days under $\mathrm{N}_{2}$ atmosphere in R.T. Finally, the resulting suspension was filtrated through PTFE membrane filter $(0.2 \mu \mathrm{m})$ and washed with dichloromethane, methanol and acetone. The solid residue was collected to obtain $16 \mathrm{mg}$ of 6 as powder.

Material 8. Material $6(15 \mathrm{mg})$ was dissolved in DMF $(15 \mathrm{~mL})$ and an excess of $\alpha$-amino acid $7(17 \mathrm{mg}$, $0.06 \mathrm{mmol})$ and formaldehyde $(8 \mathrm{mg}, 0.27 \mathrm{mmol})$ were added. The reaction mixture was heated at 120 ${ }^{\circ} \mathrm{C}$ for 3 days. Every 24 hours an excess of 7 ( $17 \mathrm{mg}, 0.06 \mathrm{mmol}$ ) and formaldehyde $(8 \mathrm{mg}, 0.27 \mathrm{mmol})$ was added. Afterwards, the mixture was filtrated through PTFE membrane filter $(0.2 \mu \mathrm{m})$, washed with DMF and dichloromethane to obtain $15 \mathrm{mg}$ as powder. Then, all material was added in dichloromethane $(20 \mathrm{~mL})$ and treated with trifluoroacetic acid $(5 \mathrm{~mL})$, under stirring overnight. After filtration in a PTFE membrane filter $(0.2 \mu \mathrm{m})$ and washing with deionized water, acetone and methanol, the filter cake was dispersed in methanol $(50 \mathrm{~mL})$. Then, triethylamine $(10 \mathrm{~mL})$ was added and the mixture was left to stir for 15 minutes. Finally, the suspension was filtered over PTFE membrane filter $(0.2 \mu \mathrm{m})$ and washed with DMF, acetone and methanol to obtain $15 \mathrm{mg}$ of 8 as powder.

3D-2D heterostructure 9. In a two-neck round bottom flask, material 8 (9 mg) was added in dry dichloromethane (15 mL) and sonicated for 30 min under $\mathrm{N}_{2}$ atmosphere. Afterwards, EDCl (3.22 mg, $0.0168 \mathrm{mmol}$ ) was added at $0{ }^{\circ} \mathrm{C}$ and the suspension was left to stir for $1 \mathrm{~h}$. Then, MoS -based material $4(3.1 \mathrm{mg})$, DMAP $(4.7 \mathrm{mg}, 0.024 \mathrm{mmol})$ and $\mathrm{N}, \mathrm{N}$-diisopropylethylamine $(20 \mu \mathrm{L})$ were added and the dispersion was left to stir for five days at room temperature. Finally, the resulting suspension was 
filtrated through PTFE membrane filter $(0.2 \mu \mathrm{m})$ and washed with dichloromethane, methanol and acetone to obtain $12 \mathrm{mg}$ of $\mathbf{9}$ as powder.

\section{Supporting Information}

Supporting Information is available from the Wiley Online Library or from the author.

\section{Acknowledgements}

This research is co-financed by Greece and the European Union (European Social Fund) through the Operational Programme "Human Resources Development, Education and Lifelong Learning" in the context of the project "Reinforcement of Postdoctoral Researchers - 2nd Cycle" (MIS 5033021), implemented by the State Scholarships Foundation (IKY). R.A. gratefully acknowledges the support from the Spanish MICINN (PID2019-104739GB-100/AEI/10.13039/501100011033), Government of Aragon (project DGA E13-17R (FEDER, EU)) and from the European Union H2020 programs "ESTEEM3" (Grant number 823717) and Graphene Flagship (881603). Partial financial support from the project "National Infrastructure in Nanotechnology, Advanced Materials and Micro-/Nanoelectronics" (MIS 5002772), which is implemented under the Action "Reinforcement of the Research and Innovation Infrastructures", funded by the Operational Program "Competitiveness, Entrepreneurship and Innovation" (NSRF 2014-2020), Ministry of Development and Investments, and co-financed by Greece and the European Union (European Regional Development Fund) is also acknowledged. TEM and XPS measurements were performed at the Laboratorio de Microscopias Avanzadas (LMA) - Universidad de Zaragoza (Spain). We thank G. Antorrena (LMA) for his help with the XPS data acquisition.

\section{References}

[1] N. Karousis, I. Suarez-Martinez, C. P. Ewels, N. Tagmatarchis, Chem. Rev. 2016, 116, 4850-4883.

[2] A. Kagkoura, N. Tagmatarchis, Nanomaterials 2020, 10, 1407. 
[3] G. Pagona, N. Tagmatarchis, J. Fan, M. Yudasaka, S. Iijima, Chem. Mater. 2006, 18, 3918-3920.

[4] M. Vizuete, M. J. Gomez-Escalonilla, J. L. G. Fierro, K. Ohkubo, S. Fukuzumi, M. Yudasaka, S. Iijima, J. F. Nierengarten, F. Langa, Chem. Sci. 2014, 5, 2072-2080.

[5] G. Pagona, A. S. D. Sandanayaka, T. Hasobe, G. Charalambidis, A. G. Coutsolelos, M. Yudasaka, S. Iijima, N. Tagmatarchis, J. Phys. Chem. C 2008, 112, 15735-15741.

[6] N. Tagmatarchis, A. Maigne, M. Yudasaka, S. Iijima, Small 2006, 2, 490-494.

[7] G. Pagona, N. Karousis, N. Tagmatarchis, Carbon 2008, 46, 604-610.

[8] C. Cioffi, S. Campidelli, C. Sooambar, M. Marcaccio, G. Marcolongo, M. Meneghetti, D. Paolucci, F. Paolucci, C. Ehli, G. M. Aminur Rahman, V. Sgobba, D. M. Guldi, M. Prato, J. Am. Chem. Soc. 2007, 129, 3938-3945.

[9] B. Devadas, C. C. Chang, T. Imae, Taiwan Inst. Chem. Eng. 2019, 102, 378-386.

[10]M. A. Lukowski, A. S. Daniel, F. Meng, A. Forticaux, L. Li, S. Jin, J. Am. Chem. Soc. 2013, 135, 10274-10277.

[11]D. Voiry, M. Salehi, R. Silva, T. Fujita, M. Chen, T, Asefa, V. B. Shenoy, G. Eda, M. Chhowalla, Nano Lett. 2013, 13, 6222-6227.

[12]A. Kagkoura, M. Pelaez-Fernandez, R. Arenal, N. Tagmatarchis, Nanoscale Adv. 2019, 1, 1489-1496.

[13]A. Kagkoura, I. Tzanidis, V. Dracopoulos, N. Tagmatarchis, D. Tasis, Chem. Commun. 2019, 55, 2078-2081.

[14]A. Kagkoura, R. Canton-Vitoria, L. Vallan, J. Hernandez-Ferrer, A. M. Benito, W. K. Maser, R. Arenal, N. Tagmatarchis, Chem. Eur. J. 2020, 26, 6635-6642.

[15]E. E. Benson, H. Zhang, S. A. Schuman, S. U. Nanayakkara, N. D. Bronstein, S. Ferrere, J. L. Blackburn, E. M. Miller, J. Am. Chem. Soc. 2018, 140, 441-450.

[16]R. Canton-Vitoria, Y. Sayed-Ahmad-Baraza, M. Pelaez-Fernandez, R. Arenal, C. Bittencourt, C. P. Ewels, N. Tagmatarchis, npj 2D Mater. Appl. 2017, 1, 13. 
[17]I. Sideri, R. Arenal, N. Tagmatarchis, ACS Mater. Lett. 2020, 2, 832-837.

[18]S. Bertolazzi, M. Gobbi, Y. Zhao, C. Backes, P. Samorì, Chem. Soc. Rev. 2018, 47, 6845-6888.

[19]A. Stergiou, N. Tagmatarchis, Chem. Eur. J. 2018, 24, 18246-18257.

[20]D. Voiry, A. Goswami, R. Kappera, C. de Carvalho Castro e Silva, D. Kaplan, T. Fujita, M. Chen, T. Asefa, M. Chhowalla, Nat. Chem. 2015, 7, 45-49.

[21]K. C. Knirsch, N. C. Berner, H. C. Nerl, C. S. Cucinotta, Z. Gholamvand, N. McEvoy, Z. Wang, I. Abramovic, P. Vecera, M. Halik, S. Sanvito, G. S. Duesberg, V. Nicolosi, F. Hauke, A. Hirsch, J. N. Coleman, C. Backes, ACS Nano 2015, 9, 6018-6030.

[22]E. X. Yan, M. Caban-Acevedo, K. M. Papadantonakis, B. S. Brunschwig, N. S. Lewis, 1T'-MoS 2 , ACS Mater. Lett. 2020, 2, 133-139.

[23]A. Kagkoura, R. Arenal, N. Tagmatarchis, Nanomaterials 2020, 10, 2416.

[24]X. Zhang, X.-F. Qiao, W. Shi, J.-B. Wu, D.-S. Jianga, P.-H. Tan, Chem. Soc. Rev. 2015, $44,2757-2785$.

[25]S. Bandow, A. M. Rao, G. U. Sumanansekera, R. C. Eklund, F. Kokai, K. Takahashi, M. Yudasaka, S. Iijima, Appl. Phys. A Mater. Sci. Process. 2000, 71, 561-564.

[26]J. Cao, J. Zhou, Y. Zhang, X. A. Liu, Sci. Rep. 2017, 7, 8825.

[27]D. Briggs, M. P. Seah, Practical Surface Analysis by Auger and X-ray Photoelectron Spectroscopy, Wiley, Chichester, NY, USA 1983.

[28]L. Alvarez, F. Fall, A. Belhboub, R. Le Parc, Y. Almadori, R. Arenal, R. Aznar, P. Dieudonné-George, P. Hermet, A. Rahmani, B. Jousselme, S. Campidelli, J. Cambedouzou, T. Saito, J.-L. Bantignies, J. Phys. Chem. C 2105, 119, 5203-5210.

[29]K. Zhang, B. Jin, Y. Gao, S. Zhang, H. Shin, H. Zeng, J. H. Park, Small 2019, 1804903. [30]J. E. Lee, J. Jung, T. Y. Ko, S. Kim, S.-I. Kim, J. Nah, S. Ryu, K. T. Nam, M. H. Lee, RSC Adv. 2017, 7, 5480-5487. 
[31]H.-Y. He, Sci Rep. 2017, 7, 45608.

[32]P. Gnanasekar, D. Periyanagounder, J. Kulandaivel, Nanoscale 2019, 11, 2439-2446.

[33]X. Yu, G. Zhao, S. Gong, C. Liu, C. Wu, P. Lyu, G. Maurin, N. Zhang, ACS Appl. Mater. Interfaces 2020, 12, 24777-24785.

[34]J. Jeanguillaume, C. Colliex, Ultramicroscopy, 1989, 28, 252-257.

[35]R. Arenal, F. de la Pena, O. Stephan, M. Walls, A. Loiseau, C. Colliex, Ultramicroscopy, 2008, 109, 32-38. 
The synthesis of 3D-2D CNH-MoS 2 heterostructures was accomplished. The increased $\mathrm{MoS}_{2}$ loading, covalently bound at the tips and sidewalls of $\mathrm{CNHs}$ and the covalent linkage between $\mathrm{CNH}$ and $\mathrm{MoS}_{2}$ facilitate electrocatalytic activity for protons reduction at heterointerfaces same to that of commercial $\mathrm{Pt} / \mathrm{C}$.

A. Kagkoura, ${ }^{1}$ R. Arenal, ${ }^{2,3,4}$ N. Tagmatarchis ${ }^{1 *}$

Controlled chemical functionalization toward 3D-2D carbon nanohorn-MoS $S_{2}$ heterostructures with enhanced electrocatalytic activity for protons reduction

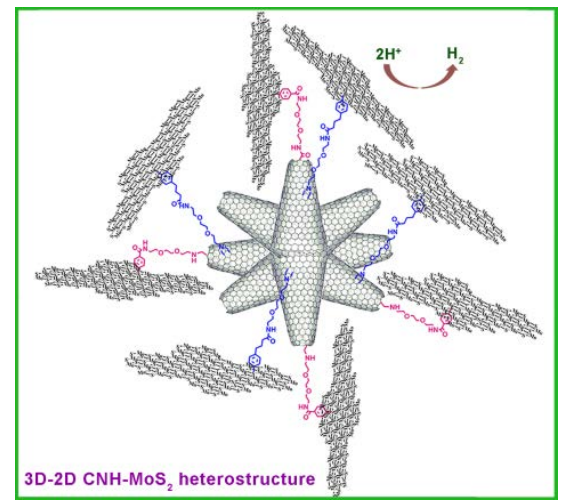


Supporting Information

Controlled chemical functionalization toward 3D-2D carbon nanohorn-MoS 2 heterostructures with enhanced electrocatalytic activity for protons reduction

Antonia Kagkoura, ${ }^{1}$ Raul Arenal, ${ }^{2,3,4}$ Nikos Tagmatarchis ${ }^{1 *}$

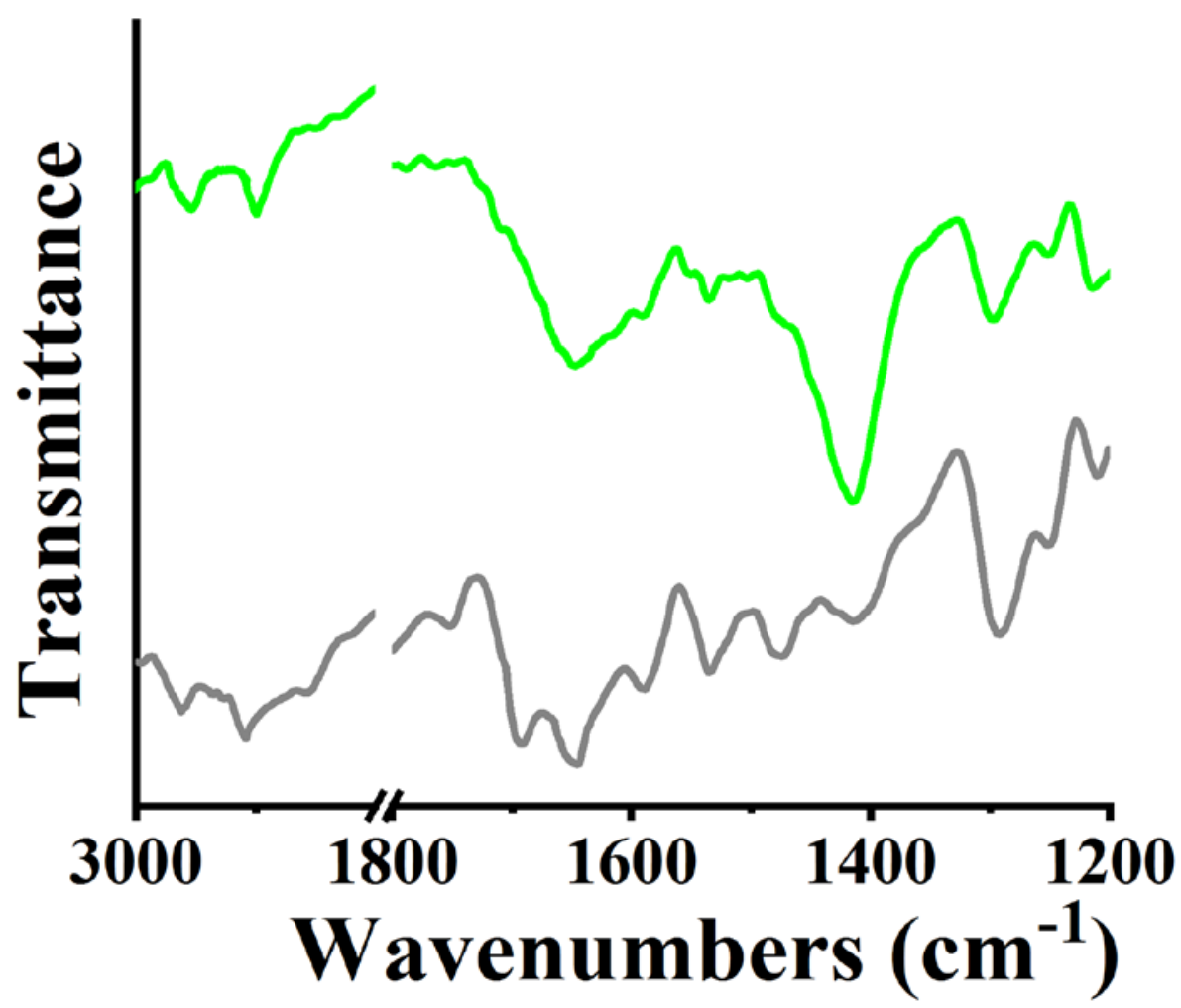

Figure S1. FT-IR spectra of BOC-modified $\mathrm{MoS}_{2}$ nanosheets 2 (grey) and amino-modified $\mathrm{MoS}_{2}$ nanosheets $\mathbf{3}$ (green). 


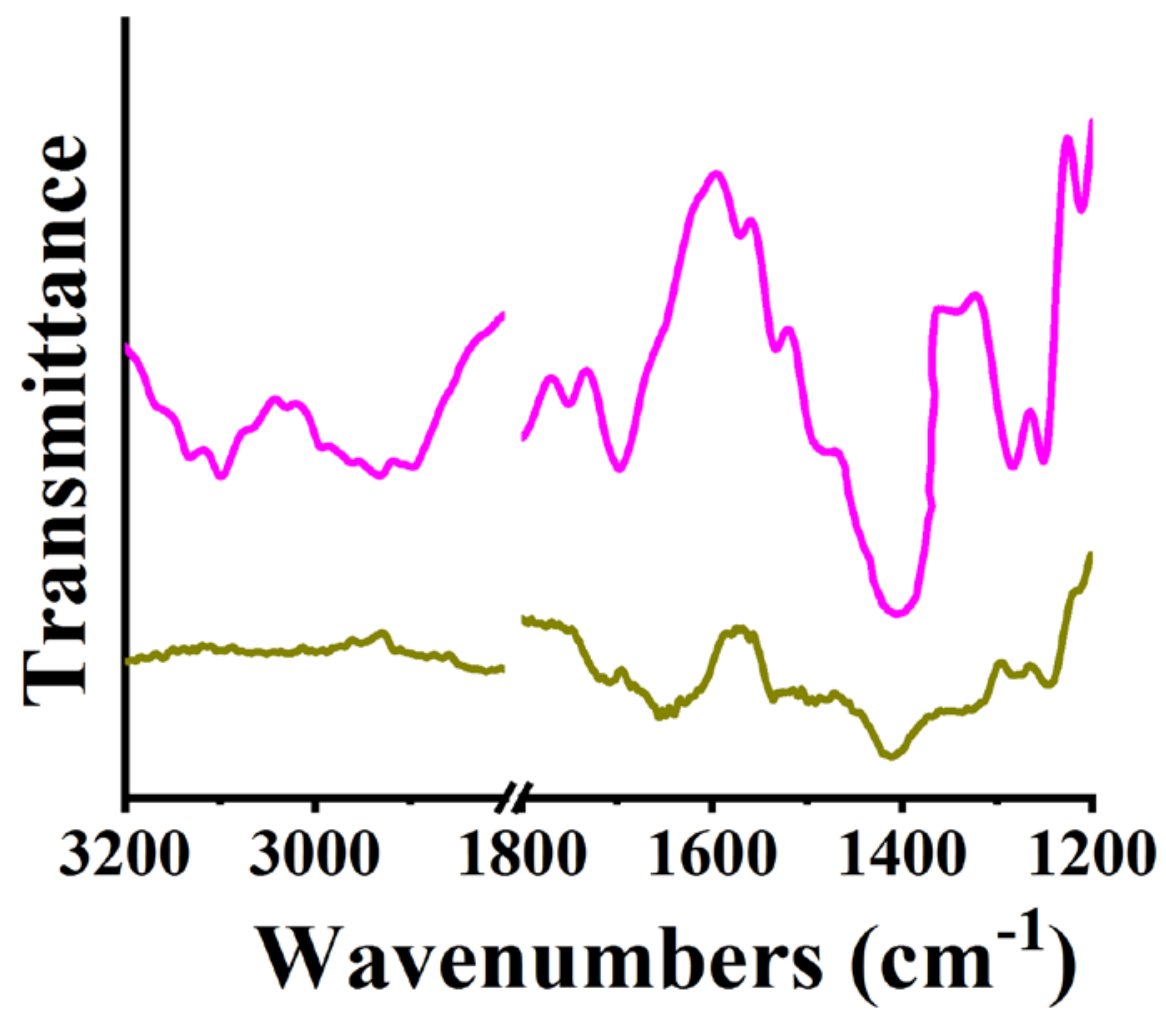

Figure S2. FT-IR spectra of oxidized CNHs 5 (pink) and pristine CNHs (yellow). 


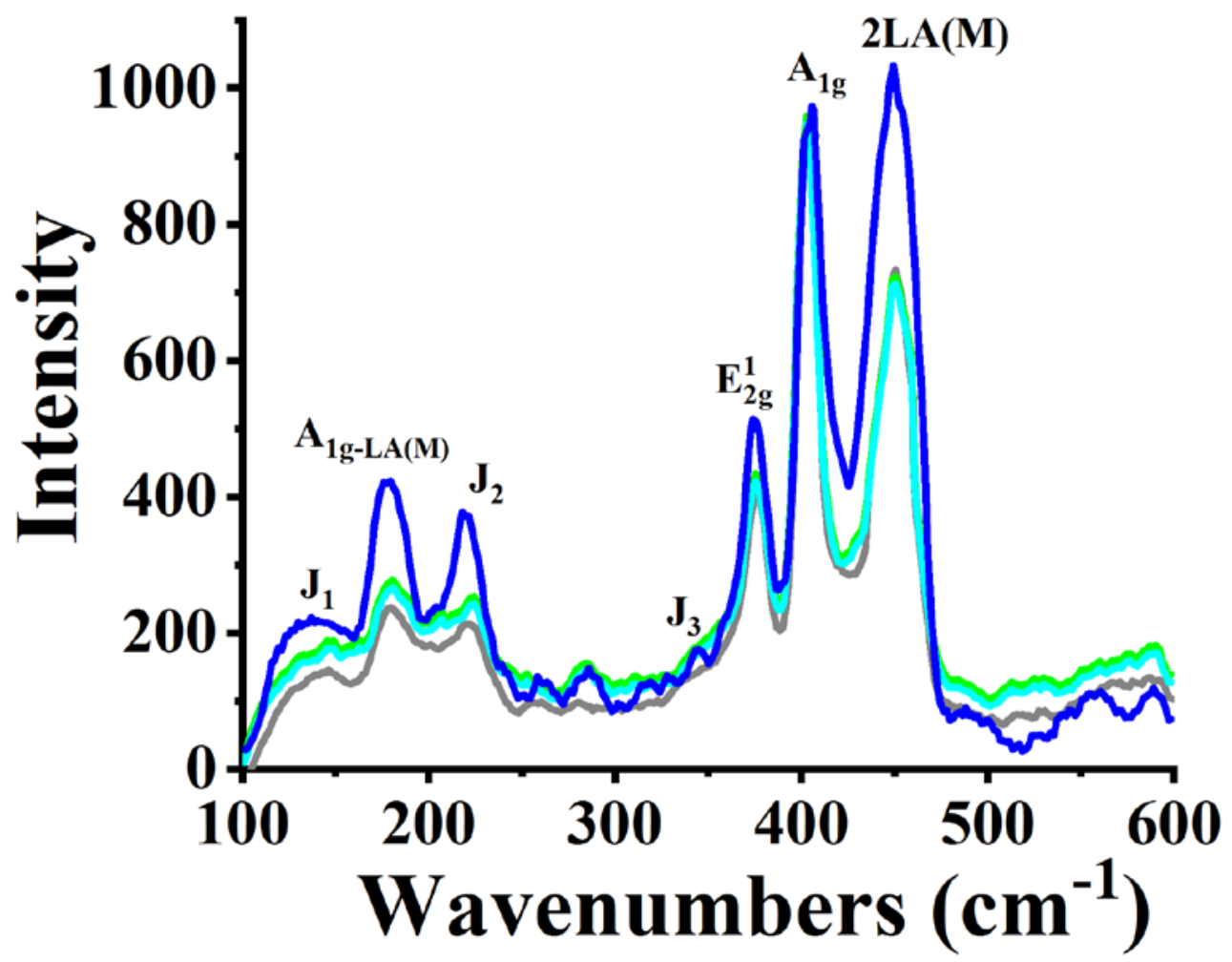

Figure S3. Raman spectra of exfoliated $1 \mathrm{~T}-\mathrm{MoS}_{2}$ (blue) and modified $\mathrm{MoS}_{2}$ materials 2 (grey), $\mathbf{3}$ (green) and 4 (cyan), recorded upon excitation at $633 \mathrm{~nm}$. 


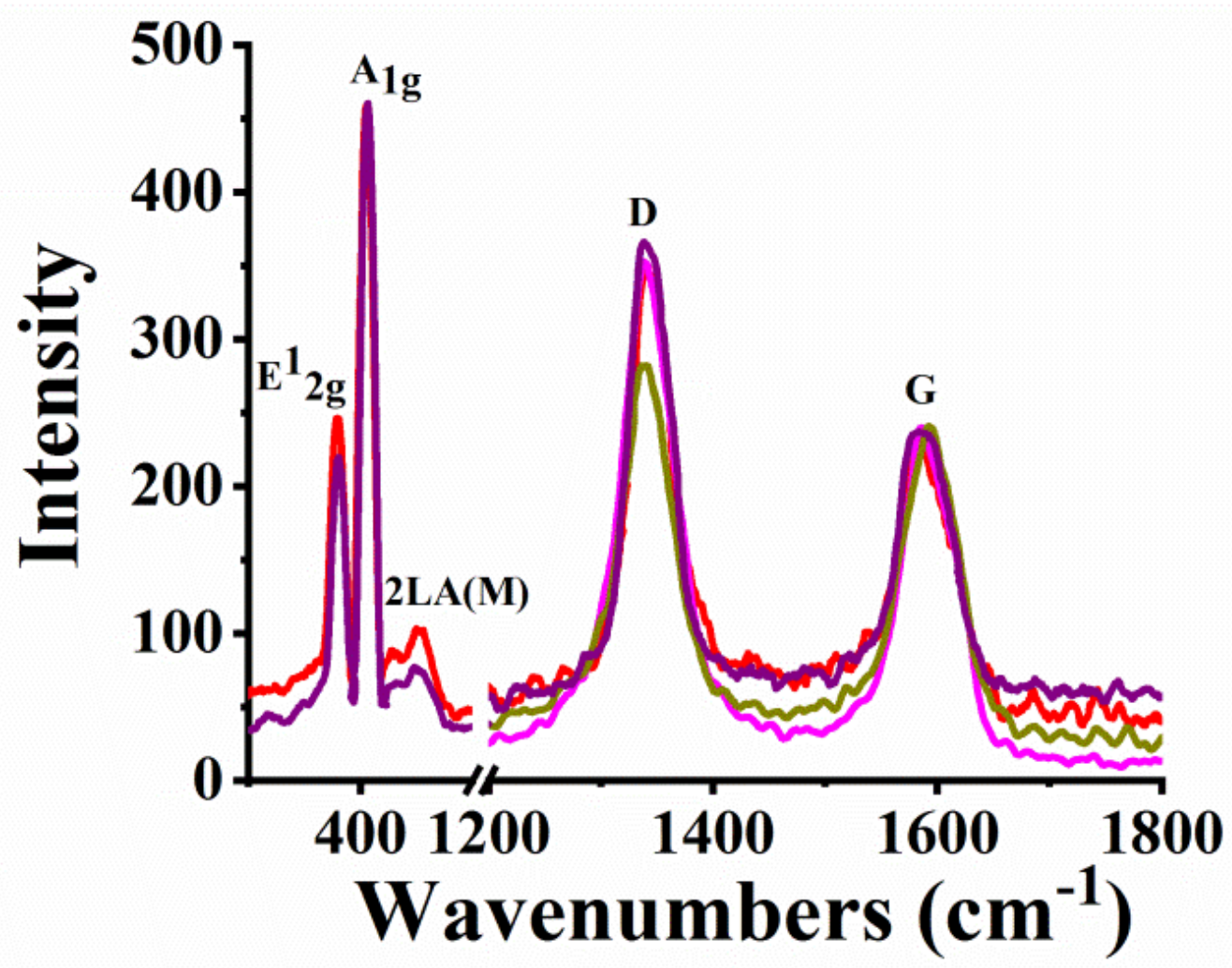

Figure S4. Raman spectra of pristine CNHs (dark yellow), oxidized CNHs 5 (pink), 6 (black) and heterostructure 8 (purple) recorded upon excitation at $514 \mathrm{~nm}$. 

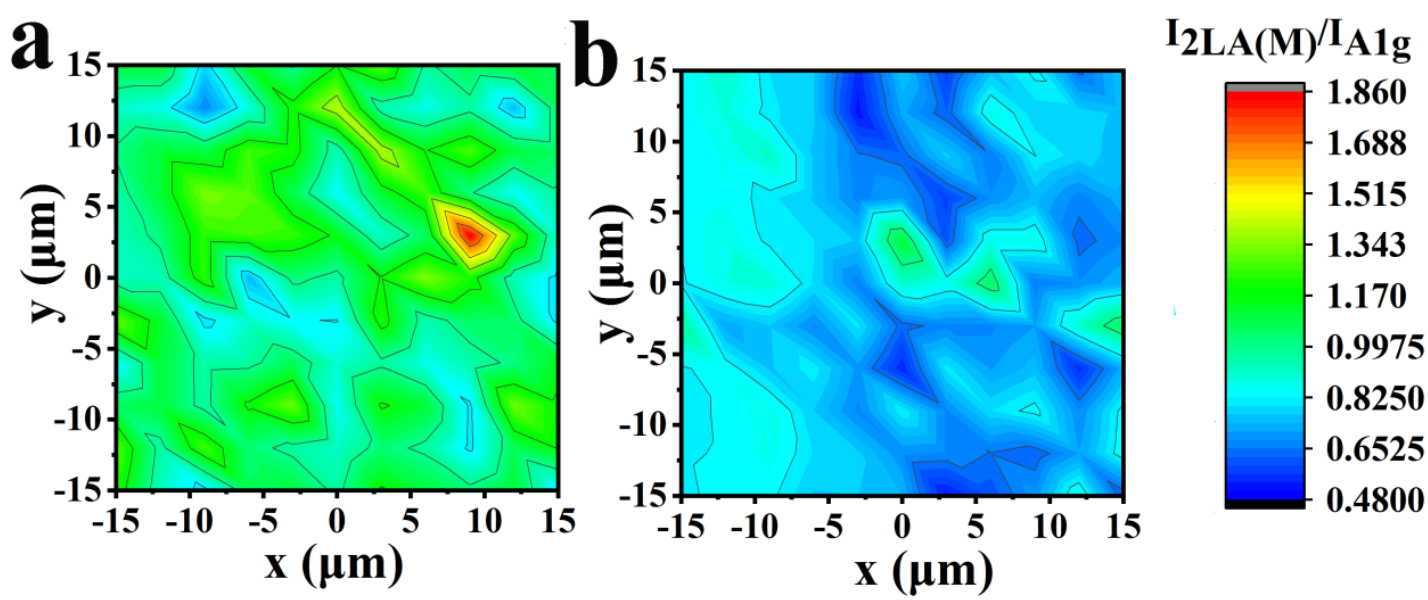

Figure S5. Raman maps of (a) exfoliated 1T-MoS and (b) modified $\mathrm{MoS}_{2}$ material 4 recorded upon excitation at $633 \mathrm{~nm}$.
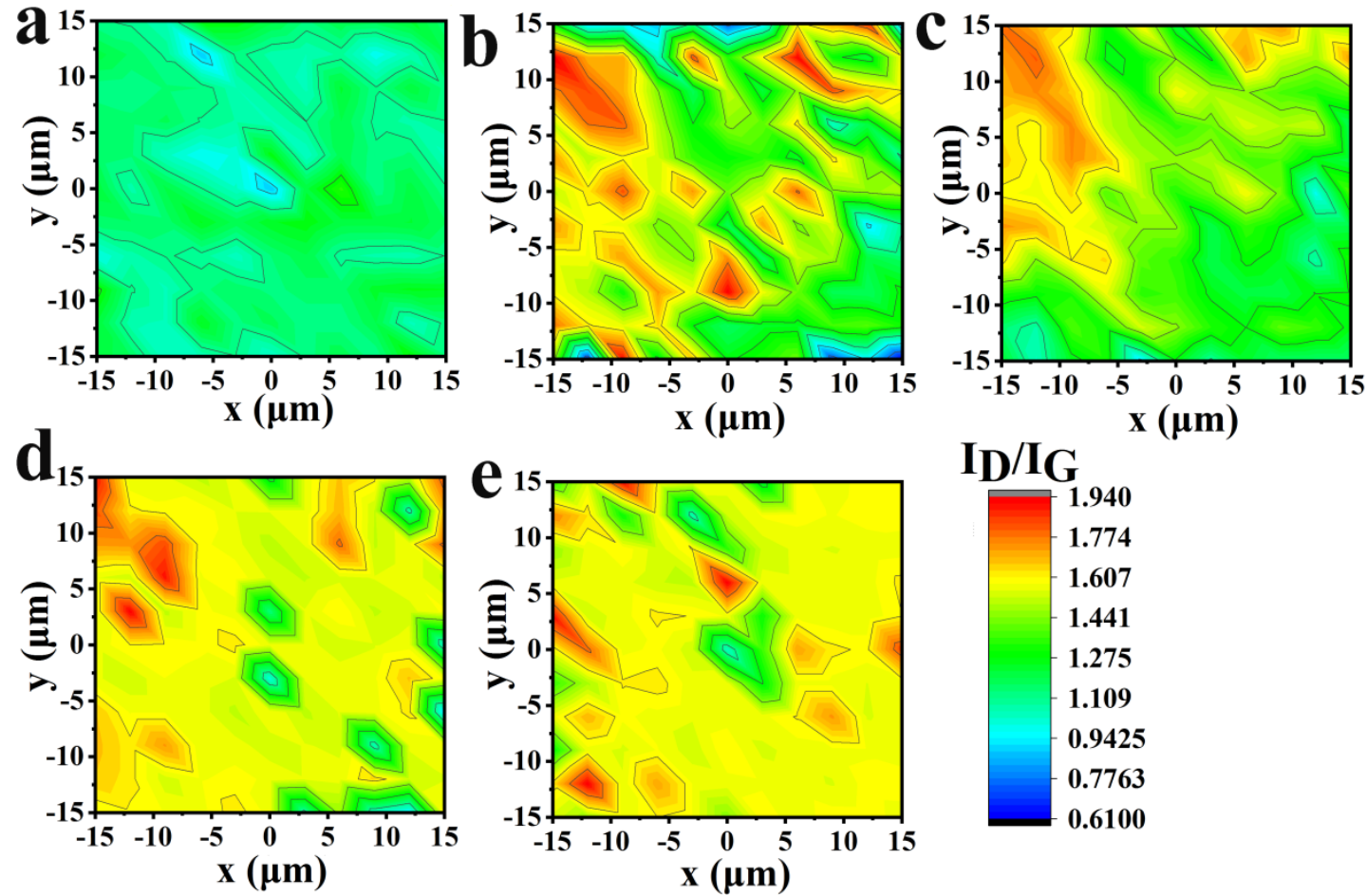

Figure S6. Raman maps for (a) pristine CNHs, (b) oxidized CNHs 5, (c) 6, (d) 7 and (e) 8 recorded upon excitation at $514 \mathrm{~nm}$. 


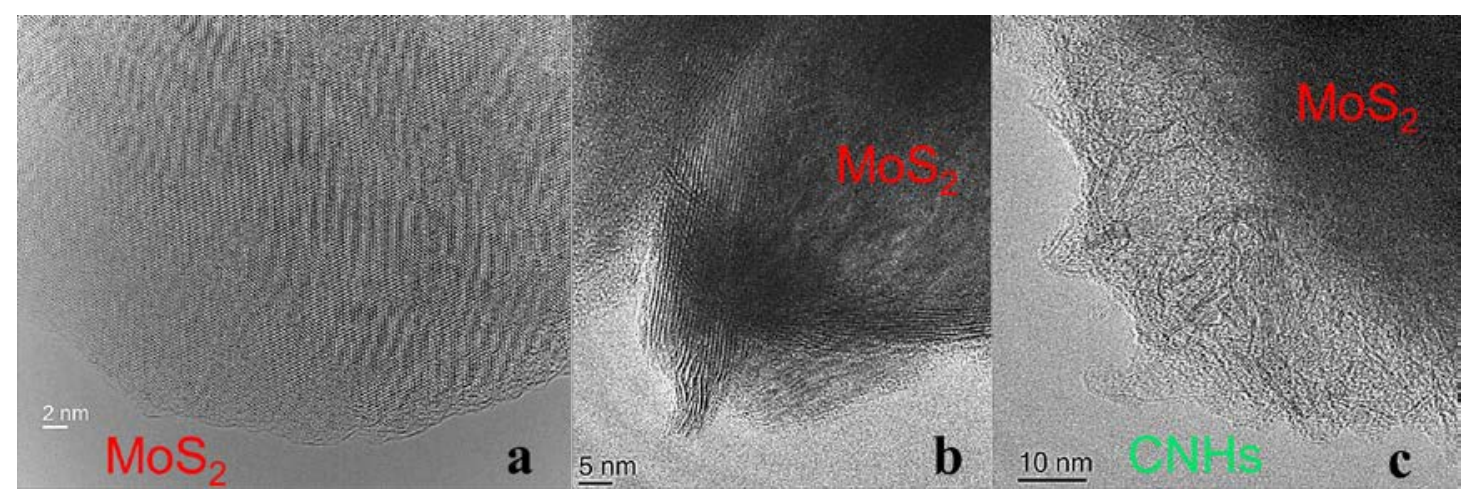

Figure S7. HRTEM image of $(a, b)$ exfoliated $\mathrm{MoS}_{2}$ flake, and $(\mathrm{c})$ heterostructure 8 showing the presence of $\mathrm{CNHs}$ and $\mathrm{MoS}_{2}$. 


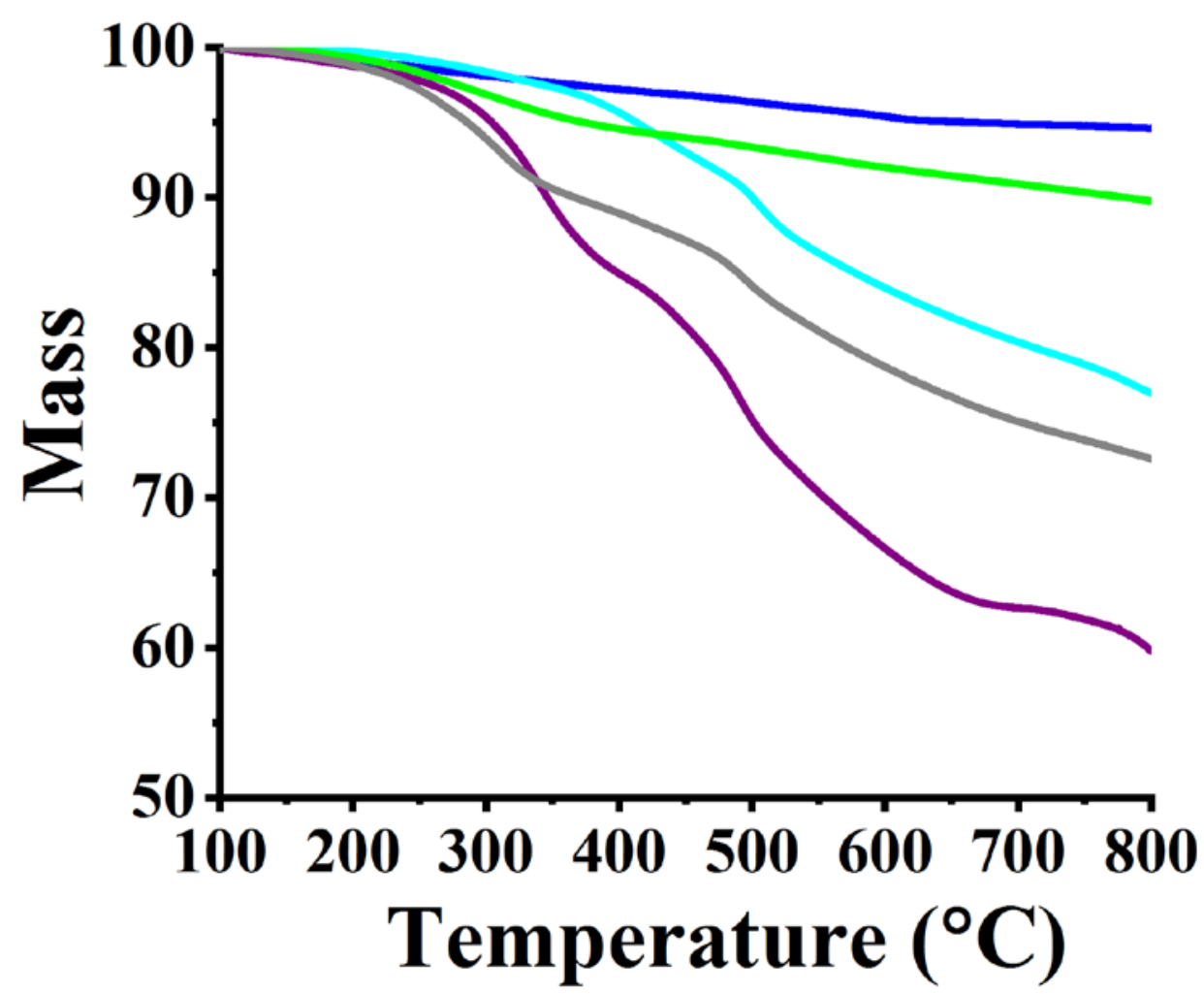

Figure S8. TGA graphs for exfoliated 1T-MoS 2 (blue), BOC-modified $\mathrm{MoS}_{2}$ material 2 (grey), aminomodified $\mathrm{MoS}_{2}$ material 3 (green), modified $\mathrm{MoS}_{2}$ material 4 (cyan) and heterostructure 8 (purple), recorded under nitrogen atmosphere. 


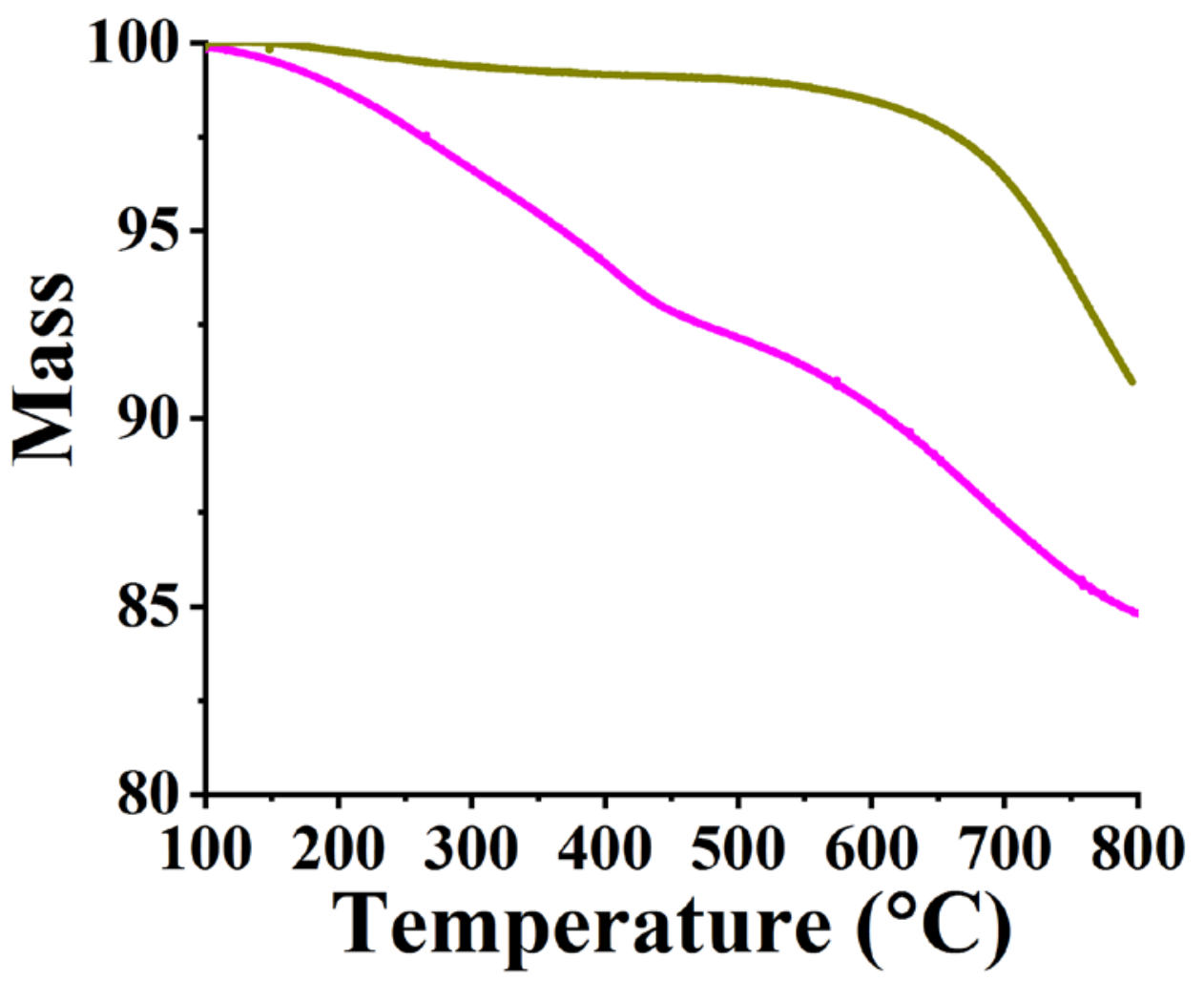

Figure 59. TGA graphs for pristine CNHs (yellow) and oxidized CNHs 5 (pink) recorded under nitrogen atmosphere. 

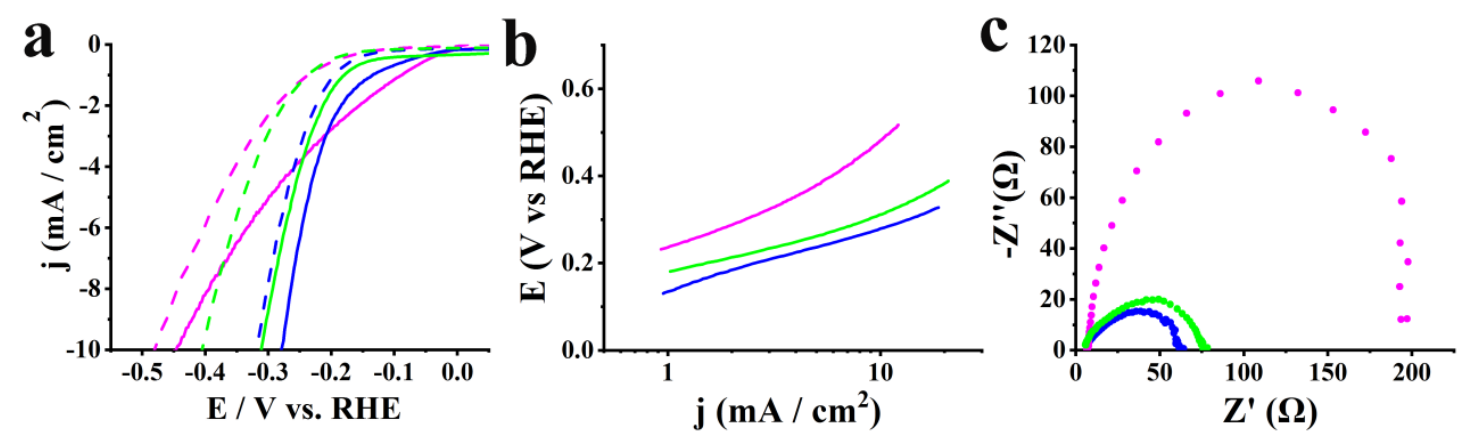

Figure S10. (a) LSVs for HER before (solid lines) and after 10,000 cycles (dashed lines), (b) Tafel slopes and (c) Nyquist plots, for exfoliated 1T-MoS 2 (blue), material 3 (green) and oxidized CNHs 5 (pink). LSVs obtained at 1,600 rpm rotation speed and $5 \mathrm{mV} / \mathrm{s}$ scan rate in aqueous $0.5 \mathrm{M} \mathrm{H}_{2} \mathrm{SO}_{4}$. 

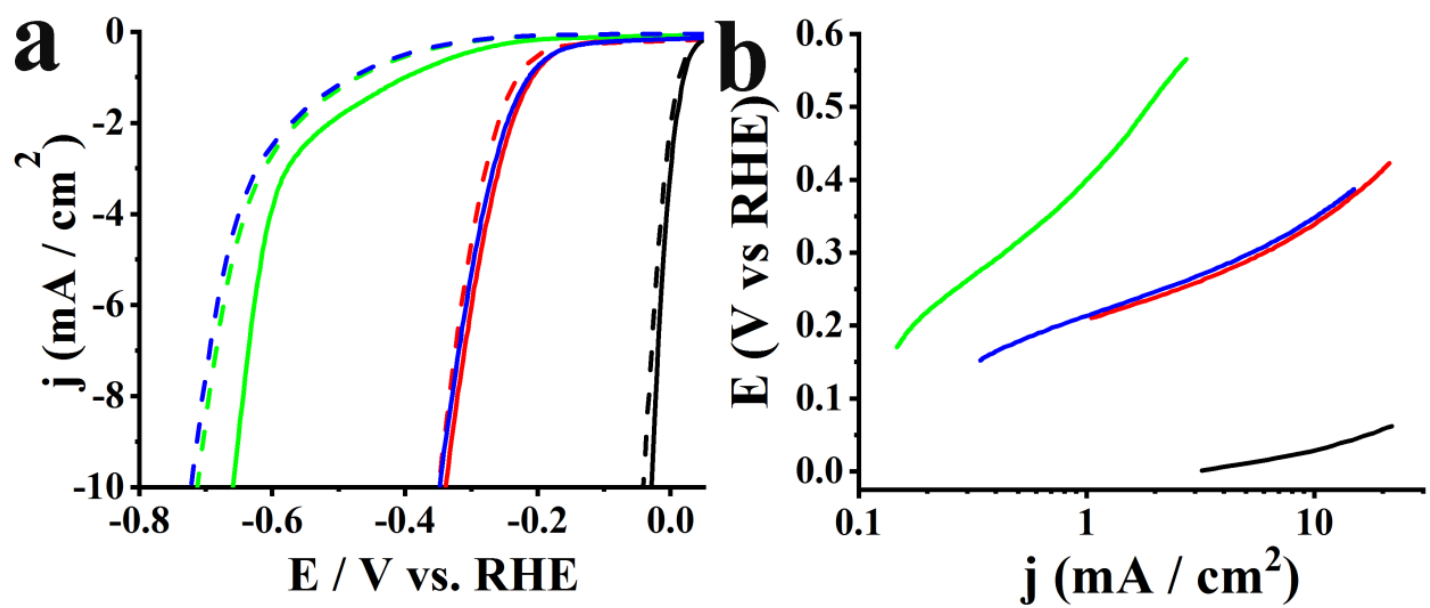

Figure S11. (a) LSVs for HER before (solid lines) and after 10,000 cycles (dashed lines) and (b) Tafel slopes for mixtures of $\mathbf{3}$ and $\mathbf{5}$ (green), $\mathbf{4}$ and $\mathbf{6}$ (blue) and materials $\mathbf{6}$ (red), $\mathbf{9}$ (black). LSVs obtained at 1,600 rpm rotation speed and $5 \mathrm{mV} / \mathrm{s}$ scan rate in aqueous $0.5 \mathrm{M} \mathrm{H}_{2} \mathrm{SO}_{4}$. 